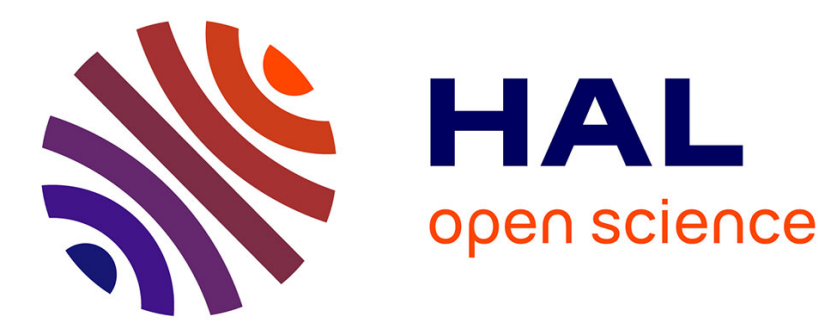

\title{
Multi criteria analyses for managing motorway company facilities: the Decision Support System SINERGIE
}

\author{
Jacky Montmain, Céline Sanchez, Marc Vinches
}

\section{To cite this version:}

Jacky Montmain, Céline Sanchez, Marc Vinches. Multi criteria analyses for managing motorway company facilities: the Decision Support System SINERGIE. Advanced Engineering Informatics, 2009, 23 (3), pp.265-287. 10.1016/j.aei.2008.12.001 . hal-00353812

\section{HAL Id: hal-00353812 \\ https://hal.science/hal-00353812}

Submitted on 26 May 2021

HAL is a multi-disciplinary open access archive for the deposit and dissemination of scientific research documents, whether they are published or not. The documents may come from teaching and research institutions in France or abroad, or from public or private research centers.
L'archive ouverte pluridisciplinaire HAL, est destinée au dépôt et à la diffusion de documents scientifiques de niveau recherche, publiés ou non, émanant des établissements d'enseignement et de recherche français ou étrangers, des laboratoires publics ou privés. 


\title{
Multi criteria analyses for managing motorway company facilities: The decision support system SINERGIE
}

\author{
Jacky Montmain $^{\mathrm{a}, *}$, Céline Sanchez ${ }^{\mathrm{b}}$, Marc Vinches ${ }^{\mathrm{a}}$ \\ a École des Mines d'Alès, 6 Avenue de Clavières, 30319 Alès Cedex, France \\ bervice Intégration et Domaines Transverses, Société des Autoroutes Estérel Côtes d'Azur Provence Alpes ESCOTA, Direction Technique, \\ 432 Avenue de cannes BP 41, 06211 Mandelieu Cedex, France
}

\section{A B S T R A C T}

Improvements to the decision-making process in the areas of preventive maintenance and facility repairs for a range of infrastructure include maintenance, upgrading and retrofitting operations. Depending on the available information concerning the condition of the facilities, specific types of projects can be pro-grammed. Assigning a priority of action to a given project necessitates an evaluation process that intro-duces several criteria at various functional levels. This multi criteria (MC) evaluation is modelled as the aggregation of partial scores attributed to a project with regards to (w.r.t.) a given set of criteria. The scores are expressed over a finite scale which can cause some troubles when no attention is paid to the aggregation procedure. This paper deals with the consistency of the evaluation process, where scores are expressed as labels by experts (over finite discrete scales), whereas the aggregation model is sup-posed to deal with numerical values and cardinal scales. This is a curious but common apparent paradox in MC evaluation when engineering contexts are concerned. Adequate mathematical tools are provided to tackle the related problems. A robustness analysis of the evaluation process concludes the mathematical part of the paper.

The mathematical choices regarding our information processing chain meet the ESCOTA motorway company requirements. Indeed, ESCOTA aims at the formalization and improvement of the decisional process for preventive maintenance of its assets in a MC framework. According to available pieces of knowledge on the infrastructure condition, projects are to be evaluated w.r.t. technical but also to con-formity, security and financial criteria for planning purposes. ESCOTA's engineers are used to manipulate symbolic labels but might convert them into more or less arbitrary numerical values when necessary without the necessary care. The SINERGIE decision support system has been developed for ESCOTA to implement a logically sound information processing methodology. The SINERGIE man-machine inter-faces illustrate the way by which the mathematical concepts described in this paper are used in terms of infrastructure management activities. The various information processing phases laid out in SINERGIE have been illustrated for road applications.

\section{Introduction}

Advanced computing applications are changing the way engineers interact with computers and many examples can be found in infrastructure management; infrastructure management itself concerns the full life cycle of the infrastructure, including the planning, design, construction, commissioning, operation, maintenance, rehabilitation and decommissioning. Engineers in charge of large-scale infrastructure management are now skilled in the craft of using computational tools for tasks such as numerical

\footnotetext{
* Corresponding author. Present address: Laboratoire de Génie Informatique et d'Ingénierie de la Production, Ecole des Mines d'Alès - Site EERIE, Parc Scientifique G. Besse, 30035 Nîmes cedex, France. Tel.: +33 4663870 58; fax: +33 466387074 E-mail addresses: jacky.montmain@ema.fr (J. Montmain), cesanchez@escota.net (C. Sanchez), marc.vinches@ema.fr (M. Vinches).
}

analysis, drafting, detail design and other aspects of project planning [1]. A lot of soft computing solutions have been proposed in the recent years to assist them in any step of the life-cycle of an infrastructure from design to maintenance. Thus, the impact of information and communication technologies (ICT) is a kernel factor in developing our modes of organisation, if not of our societies. Regardless of how humans are involved in systems nowadays, the systems are so complex that increasingly intricate and inescapable dynamic information processing systems are bound to emerge. Decision support systems (DSS) constitute one of the most significant classes of ICT tools and have been the subject of thorough investigations. DSS may rely on statistical, data-mining, knowledge discovery techniques, but also on operational research, theories of uncertainty and multi criteria techniques. The next paragraph gives a short panel of some works relative to decision-making aids in the area of infrastructure management. 
Saridakis et al. [2] survey the application of soft computing techniques in engineering design. Within this context, fuzzy logic, genetic algorithms and artificial neural networks, as well as their fusion are reviewed in order to examine the capability of soft computing methods and techniques to effectively address various hard-to-solve design tasks and issues. In [3], the authors explain that infrastructure managers rely on capabilities of computeraided design (CAD) and geospatial information systems (GIS) for making decisions during the implementation of engineering tasks. Engineers in infrastructure management must gain knowledge and skills in both CAD and GIS to perform these tasks. Interoperability is at the heart of their work and is seen as a solution to overcome the problems associated with heterogeneous environments. The interoperability may occur at different levels and for different purposes. In a recent paper, Dehlin and Olofsson [4] discuss the motivation to innovate and to introduce new ICT tools and working methods into the construction industry. In view of this, they explain that a new project-oriented evaluation model is developed with the purpose of providing for a structure and a work routine to be used by a multidisciplinary project team to evaluate the implications of realizing ICT investments in construction projects. They underline that new ICT tools in construction have to support sharing of information and give guidelines to evaluate their value or impact on the profitability of the project. Although primarily aimed at establishing future benefits and costs, their model may be used for follow-ups. Kostoulas et al. [5] highlight the role of ICT in construction project control. Integrating promising information technologies such as radio frequency identification (RFID) technology, mobile devices-PDA and web portals can help improve the effectiveness and the convenience of the information flow in the construction systems to control the supply chain. More marginally, Kostoulas et al. [6] present a decentralized trust model to enhance reliable information dissemination in large-scale disasters relief operations involving civil engineers.

Decision support systems (DSS) constitute probably the most cognitive ICT tools. A DSS is defined as the combination of data, information, and computer based tools and services working within a structured framework to improve the process and outcome of decision-making [7]. Vanier Dana [7] mentions six characteristics of a DSS: (1) explicit design to solve ill-structured problems; (2) powerful and easy-to-use interface; (3) ability to flexibly combine analytical models with data; (4) ability to explore the solution space by building alternatives; (5) capability of supporting a variety of decision-making styles and (6) allow interactive and recursive problem-solving. In the special issue in ITCON, DSS in infrastructure management [7], Vanier introduces a series of papers that illustrate the role of DSS in infrastructure management. Computer based tools play a key role: 3D visualization tools to plan and lay out urban green spaces, geographic information systems (GIS) [8], ontologies and more generally semantic web to promote interoperability in large scale integrated projects $[9,10]$, knowledge based decision support system $[11,12]$, user-friendly man-machine interface [13], planning software [13], client-server decision support architecture for project management [14].

Many water utilities are faced with the problem of ageing pipe networks and the associated increasing costs. That is why lots of DSS have been proposed in this field since the sixties. [15] presents a decision support system called PARMS-PRIORITY, a software application to support decisions regarding pipeline renewal prioritisation. The modules described are based on key decision-making tasks, such as: risk calculation, failure prediction, costs assessment, data exploration and scenario evaluation. In the same way, a method for estimating water network rehabilitation needs is proposed in [16]. [17] also proposes a DSS for infrastructure maintenance to water supply systems. Finally, [18] proposes a DSS for rehabilitation planning and optimisation of the maintenance of underground pipe networks of water utilities: the DSS performs reliability based life predictions of the pipes and determines the consequences of maintenance and neglect over time in order to optimize a rehabilitation policy. The aim of a DSS may also be to satisfy the high transparency requirement for financial controls and the information demand for forward financial planning [19-21]. The techniques behind these works are most often data mining, neural networks [21] and signal processing, e.g. data compression, data streams, noise filtering, from one hand [22], multi attribute utility theory and operational research (optimization problems with discrete and/ or continuous variables) from the other hand [12]. [23] stimulates interest within the civil engineering research community for developing the next generation of applied artificial neural networks. Data-mining, knowledge discovery and automated learning techniques are of use in many civil engineering activities. Multi criteria approaches also provide relevant tools in civil engineering. For example, [24] concerns design governed by multiple objective criteria, which are conflicting in the sense of competing for common resources to achieve variously different performance objectives (financial, functional, environmental, esthetical, etc.). A multi criteria decision making (MCDM) strategy is proposed that employs a trade-off-analysis technique to identify compromise designs for which the competing criteria are mutually satisfied in a Pareto-optimal sense.

Decisions concerning infrastructure management thus include many facets, viewpoints, e.g. the techniques to employ, current regulations, the impact on user safety, and operating costs, all within a changing social context. This problem raised in assisting decision-makers is therefore essentially multi criteria in nature and dependent upon technical, regulatory, safety and financial aspects. Decisions such as these involve comparing alternatives that have strengths or weaknesses with regard to multiple objectives of interest to the decision maker. Multi-attribute utility theory (MAUT) is a structured methodology designed to handle the tradeoffs among multiple objectives. Utility theory is a systematic approach for quantifying individuals' preferences. It is used to rescale a numerical value on some measure of interest onto a $0-1$ scale with 0 representing the worst preference and 1 the best. This allows the direct comparison of many diverse measures. MAUT can be applied in multidimensional assessments of infrastructures for maintenance or rehabilitation purposes $[25,26]$. One of the first applications of MAUT involved a study of alternative locations for a new airport in Mexico City in the early 1970s. The factors that were considered included cost, capacity, access time to the airport, safety, social disruption and noise pollution. As an example, Smith et al. [27] explain that the role of infrastructure management has been continuously changing since the late 1980's. Indeed, public agencies have started incorporating private sector practises. These new practises include using customer inputs to develop new goals and policies, developing new evaluation procedures for priority programming optimization, and adding feedback loops into infrastructure management systems. One of the new MAUT-based evaluation procedures that has been adopted into infrastructure management is the analytic hierarchy process (AHP). The AHP is a decision making-tool that incorporates both qualitative and quantitative factors. The AHP has increased in use and popularity due to the process reflecting the way people think and make decisions by simplifying complex decisions into a series of one-on-one comparisons [28]. A methodology for prioritising between different maintenance actions in the railway infrastructure is presented in [29]. The consistency of the prioritisation and the feasibility of the applied methodology are investigated. Criteria describing the diverse effects of maintenance are developed and presented to railway systems managers, together with a set of maintenance actions that are specific for each manager. Then, the analytical hierarchy 


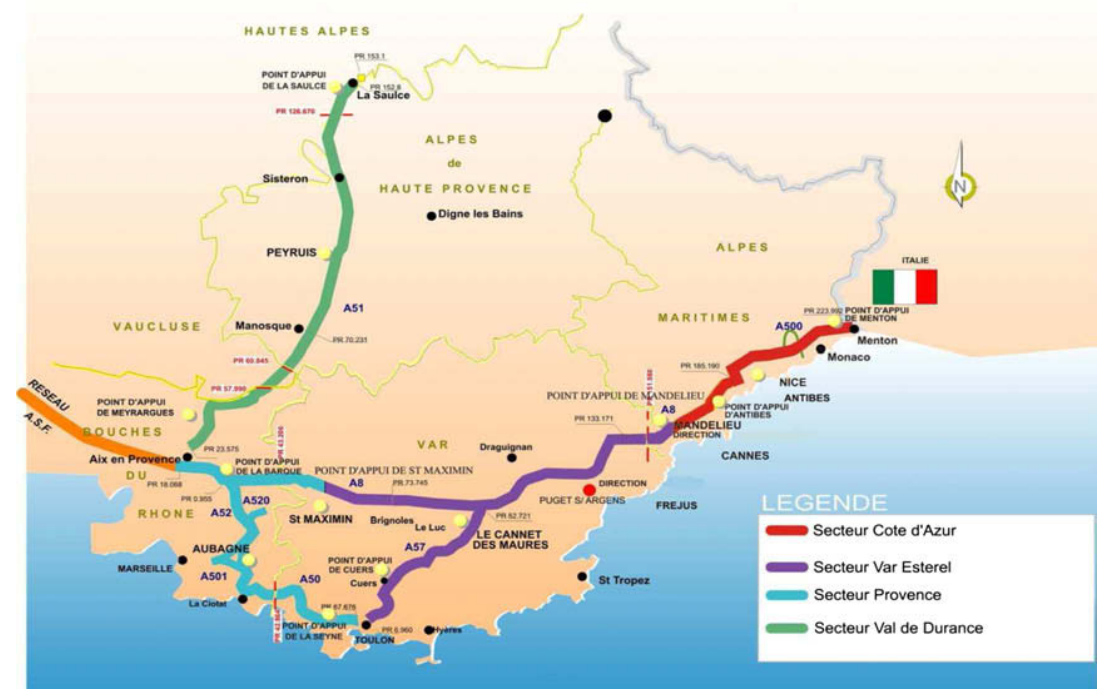

Fig. 1. ESCOTA motorway network.

process (AHP) is used to obtain preferences for the criteria and for the different actions. MAUT has also been used in more strategic decision-making modelling such as a dispute resolution selection model prototype for international construction in [30] or a model for the selection of the critical analysis method of construction proposals in a multi actors' context [31].

Our work deals with MAUT-based multidimensional assessments of infrastructures for maintenance or rehabilitation purposes. The French Public Works Ministry coordinates research projects in the field of engineering infrastructure, particularly within the scope of missions carried out by the Ministry Civil \& Urban Engineering Research Network (French acronym: RGC\&U). The second point in the 2005 call for research and innovation projects, organized by the French National Research Agency, under the banner "conservation and evaluation of existing facilities", clearly reveals that decision aid and facilities development constitute key components to the R\&D program. Various sponsors of this particular research orientation have set forth an approach built around three distinct and complementary strategies: measurement, evaluation and decision-making. Actors in the area of facility diagnostics and instrumentation (whether researchers or practitioners) are seeking a set of efficient techniques to better identify, qualify and quantify the condition of materials and their flaws. The expert, working as a technical assistant to the project owner, is required to establish instrumentation and assessment strategies while providing a diagnostic and, as such, is seeking access to useful data and procedures in order to generate a reliable diagnostic of the facility. The project owner must ensure that the facility fulfils its functions at optimal cost, within the planned operational framework, and must possess reliable input to enable him to make decisions relative to facility maintenance and repairs, or any necessary structural reinforcement. The various actors are successively involved at three levels: measurement, evaluation, and decision-making. Our works take place in this large-scale project. Our DSS supports the processes of rehabilitation planning and optimisation of the maintenance for the French motorway company ESCOTA.

On January 17, 1956, France first semi-public motorway company was created: ESCOTA (French acronym for the Esterel-Riviera Coast Motorway Company), which today has become a VINCI Concessions ${ }^{1}$ Group company, whose network coverage is displayed on

\footnotetext{
${ }^{1}$ VINCI Concessions is Europe's leading operator of transport infrastructure concessions: motorways, bridges and tunnels, car parks and airports. The acquisition of ASF and ESCOTA in 2006 was part of the group strategic plan.
}

Fig. 1. The ESCOTA Company has over the past several years built an organization and the accompanying skills to handle all three of these aspects: measurement, evaluation and decision-making. Regarding the measurement and evaluation of the condition of its infrastructure, which comprises 460 kilometres of motorway network comprising 818 bridges, 40 tunnels and 871 operation buildings, the district territorial organisation and ESCOTA Operations Division units strive to ensure that the state of knowledge with respect to facility elements is regularly updated. As for the research efforts associated with the decision-making function, the ESCOTA company decided in 2004 to install a computerized DSS SINERGIE; this system was based on the will for transparency in decision-making, in addition to transferring responsibility to its personnel at each level of intervention and justifying its decision rationale within a multi criteria and multi-actor context [32-34].

Human-computer interaction and the social implications of computer technology are at the heart of our approach which is supported by a MAUT-based DSS. It first consists in developing models that are predominantly compatible with cognitive modes used by human beings when confronted by a complex situation. Secondly our approach should acknowledge the relations that man or rather an organised group of individuals, establishes with its information system when carrying out actions and making decisions. The research efforts thus insists on knowledge management processes, collective learning and decision-making in the ESCOTA organisation, and then on what sort of information is circulated in such a socio-technical system and how it is approved. A model that explicitly supports the information processing related to the preventive maintenance decisional process of the ESCOTA motorway infrastructure is proposed. This formalization aims at breaking down the barriers existing among actors in ESCOTA through a model of human processes for handling an ill-structured cognitive task: evaluating the ageing motorway infrastructure and identifying the adequate preventive maintenance operations in the Escota organisational hierarchy. Breaking down project evaluation in terms of diagnostic, urgency and priority constitutes a vital information processing phase that facilitates and ultimately prepare a well - substantiated project plan. The multi criteria approach we propose towards infrastructure ageing assessment and decision assistance increases the level of realism and clarity provided to the decision-maker. The aggregation model we propose captures knowledge about the preferences system of ESCOTA's experts and managers. From a man-machine viewpoint, 1) our decisionmaking system mainly supports the justification of Escota decision 
strategy and traces the rationale behind decisions linked to facility management policy utilizing technical, regulatory, safety and financial elements. Indeed, we believe decision elucidation plays a key role in the decision acceptability [35,33]. That is why our decision-making aid system not only supports the decision-making process itself but also proposes knowledge management opportunities that provide explanations and diagnoses compatible with the cognitive modes of human beings. The ability of a decision support system to justify a decision strategy is an expectation of managers, the DSS must be seen as a recommender system [36-39]; 2) based upon statistical considerations we also propose robustness analysis techniques of our multi criteria decision-making model that makes ESCOTA engineering decision-making more reliable: the evaluation errors inherent to the infrastructure condition assessments are used to control the decision process and highlight the interactions users can have with the DSS. Indeed, as soon as, an expert doubts the accuracy of an evaluation concerning the condition of the network assets or the manager suspects the accuracy of the diagnosis proposed by the expert on a particular element of the network, further inspections or advanced technical measurements, more quantitative and precise investigations are required to get a more reliable re-evaluation. This refinement of the infrastructure knowledge base is thus controlled through the ESCOTA policy priorities.

This paper is thus a dual-purpose presentation. The ESCOTA Company aims at the formalization and improvement of the decisional process for preventive maintenance in a MC framework. The first purpose is thus to present the main functionalities of the DSS developed to meet the motorway operator requirements. Our mathematical choices have thus been guided by the application constraints. The functionalities of the software are highlighted through a complete and comprehensive example all along the paper. Nevertheless, these functionalities rely on mathematical models that are not of common sense in civil engineering. Part of them refer to well known approaches in the MAUT, the knowledge discovery (KD) or the operational research (OR) communities. They have been adapted or extended to tackle ESCOTA's specific requirements-we discuss in particular the case of assessments over finite scales in an aggregation procedure; then, they have been integrated into a consistent information processing chain to formalize the whole decisional process for preventive maintenance. The cohabitation and completion of mathematical tools for decisionmaking in a unique DSS was a major challenge in our approach. The second purpose of the paper is thus to present this consistent and original data processing chain and its associated mathematical models that support the ESCOTA decisional process for preventive maintenance. Some computations or algorithms as the ESCOTA's strategy elucidation, the MAUT-based ageing infrastructure assessments over finite discrete scales or the risks assessments procedures are original methods; the originality of other computations is related to their integration in a wholly consistent data processing chain (for example the Macbeth method is only a first step in our discrete scales assessment of the infrastructure condition).

The paper is organized as follows. Section 2 proposes a brief description of the multi criteria hierarchical decisional process for maintaining and managing ESCOTA infrastructure facilities. The main concepts and definitions are introduced. The management of infrastructure facilities at ESCOTA presents all the characteristics of an organisational decision-making process. Section 3 is dedicated to the mathematical aspects of our work, in particular the multi criteria aggregation scheme. Some considerations are given about the way continuous cardinal scales are constructed with the ESCOTA operating domain experts. Then, we present how to build a weighted arithmetic mean (WAM) aggregation operator w.r.t. each operating domain, in order to be consistent with the identified scales. The MACBETH method is the support of these first two steps. The problem related to the finite scales, that the experts use when assigning partial scores to a project, is then considered. A method is proposed to ensure a logically sound interface between symbolic assessments and numerical computations in the framework of WAM aggregation. Section 4 is first devoted to a justification procedure of the ESCOTA operation planning policy. Secondly, section 4 proposes a sensitivity analysis to determine the potential causes of overestimation or underestimation in the evaluation process of a project. The risk of making a wrong assessment w.r.t. a project is examined to decide whether undertaking more quantitative and deeper analyses relatively to this project is worth or not. This risk examination provides the criteria that can be considered as the most probable causes for a wrong estimation: this gives indications about further investigations to be carried out. The various information processing phases laid out in SINERGIE are illustrated for road applications, several SINERGIE man/machine interfaces are provided. ${ }^{2}$ In this paper, we will consider an illustrative example of the A808 highway. ${ }^{3}$ The functionalities of the software are thus highlighted through a fictitious, but complete and comprehensive example all along the paper. Finally, the advantages and the limits of our approach are discussed.

\section{Maintenance and management of ESCOTA infrastructure facilities: a hierarchical multi criteria process}

\subsection{Characteristics of ESCOTA decisional process for the maintenance of infrastructure}

Responsibility for managing engineering facilities consists in ensuring the durability of structures and preserving its quality in order to offer users a high level of service in terms of safety and comfort. This management effort is manifested by very close monitoring and proceeding with regular maintenance, improvements and enhancements introduced on network assets (NA). All NA components are associated with a specific "facility management field".

The five facility management fields consist of:

- buildings;

- road surfaces, fencing, horizontal road markings, embankment supervision;

- fire prevention, bush control, vertical markings;

- bridges, tunnels and retaining walls;

- environment, water basins, recreational open spaces, drainage, reservoir systems; and

Responsibility for each field is assigned to a designated expert. The activities of these experts are coordinated by the infrastructure manager.

The meanings of a few key terms and expressions are first presented.

Network assets (NA): This term refers to a structure, building, etc. at a given road coordinate (RC) or over a zone defined by an interval of road coordinates.

Examples include: the road surface between road coordinates 145 and 157 (i.e. between Les Adrets and Mandelieu ramps), and the Pessicard Tunnel at road coordinate 196.

Project or operation: A project, or operation, describes works devoted to maintenance, retrofitting or upgrading, to be conducted on one or more network assets; it is associated with a specific facility management field.

\footnotetext{
2 SINERGIE Man/machine Interfaces are in French. Consequently, the main items, notes, etc. on each figure in the paper are translated into English (filigree boxed piece of text).

${ }^{3}$ Highway A808 is fictitious for confidentiality reasons.
} 
Levels of urgency and priority: Any project evaluation entails risk analysis. On the basis of measured technical data, the individuallyassigned expert performs a technical risk analysis and matches a level of urgency with each project. The facility manager then carries out a strategic risk analysis that associates a degree of priority with the particular project.

Operations management and planning constitute a complex task for decision-makers, who must cope with a tremendous amount of information. Decision-makers require a comprehensive view of the facilities in terms of both objectives and constraints, which means being able to rely upon a continuous evaluation of the NA within a dynamic operational context. It was thus decided to introduce a DSS to assist them in defining and implementing an optimized facility management strategy built around a continuous evaluation of the NAcondition, as well as of the urgency and priority of the induced set of operational tasks. The decision-makers must include several aspects into the decision, i.e.: the techniques to use, current regulations, the impact on user safety, and operating costs, all within a changing social context. The problem raised in assisting ESCOTA decision-making is therefore essentially multi criteria in nature and dependent upon technical, regulatory, safety and financial aspects.

A common issue emerges from all these works referenced in introduction: an adequate information processing seems to be the main concern to make the right decisions. That is the case of preventive maintenance at ESCOTA. The questions are: what is the information role in decision-making? What is the relationship between information and decision? How is organized the decisionmaking process? How is the memory of preventive maintenance monitored?

A critical examination of the actual decision-making process shows that a decision is not a precise, clearly identifiable act. This process is constructed, negotiated and follows an irregular path over time $[40,41]$. According to a first rational model often associated to Operational Research, the decision should be the result of a comparative selection among the various possible solutions. The decision makers and their advisers should carefully assess the risks and probable outcomes of each option, weight their advantages and drawbacks, and ultimately select the most cost-effective solution. In terms of rational calculus, this analysis postulates the existence of a single actor who acts according to a hierarchy of preferences ordered according to their utility. This attractive theoretical model completely ignores the organizational aspects that are often implicit in the decision-making process.

Let us consider the specifications regarding the organizational decision. They were initially introduced by the economist H.A. Simon [42]. The decision-making process relative to managing the set of ESCOTA facilities displays many of the characteristics associated with organizational decision-making as described by H.A. Simon.

The foundations of his theory rest on the following remarks:

- The decision-maker does not in fact possess perfect knowledge of the situation, from which the term "limited rationality", introduced by Simon, is actually derived. These limitations in the knowledge of facts and hypotheses stem primarily from constraints at the organizational level, which help select or support one scenario over others based on organizational interests [43].

- The limited capability of human beings to effectively process the full range of information flows, required as decision-making input with all their inaccuracies, uncertainties, incompleteness and even contradictions, would indicate that an efficient decision aid tool entails high-performance information processing systems.

- Once again according to Simon's model of limited rationality [44], the decision-maker is obviously tempted to orient the decision process towards a single-criterion approach, thereby ignoring the complexity of reality. The multi criteria approach towards decision assistance overcomes this restriction by increasing the level of realism and clarity provided to the decision-maker [45]. Building a model explicitly based on several criteria reflects and formalizes a natural and intuitive reasoning mode when faced with decision-making consisting in separately analyzing each consequence [45]. Different mathematical schemes are used for combining pieces of information in order to draw the decision [46].

- The various decision-making phases are not sequenced in a linear manner, but rather in loops. Many iterations are necessary, given the relatively limited information processing capacities demonstrated by man coupled with the complexities involved in any decision-making problem, before being able to adopt a definitive decision. According to the information design choice and review (IDCR) model developed by H.A. Simon, the phases of intelligence gathering, design, choice and review are intertwined without necessarily any apparent pre-established chronological rationale (Fig. 2). A formal cybernetic interpretation of this IDCR model has been provided in [33].

Our approach only refers to original and basic notions introduced by Simon: we do not claim to provide a new human sciences model for organizational decisions; we only propose mathematical and soft computing solutions in accordance with key principles introduced by the economist sixty years ago. Many human sciences researches concerning decision making have followed the work of Simon, but it is probably the last one to give this key role to information processing and soft computing to support a decision process that still obeys a plan of actions at least partially. Maintenance operations programming is a decisional process that matches this viewpoint. This concept of pre-established plan has then disappeared in the works of Suchman concerning purely organisational decisions [47].

The facility management function with the ESCOTA company falls within this category of decision-making processes, and the present analysis justifies the technical choices adopted. As such, the SINERGIE program must be able to perform the following:

- breaking down the barriers existing among actors focused uniquely on measurements, evaluation or decision-making, as these barriers adversely affect the ability to build priorities for implementing project operations while ensuring that information gets shared and properly circulated;

- computerizing the management of all data relative to NA condition, along with an automated processing feature, with the aim of scheduling projects;

- incorporating multiple evaluation criteria specific to each field of expertise, at each functional level of decision; and

- repeating the measurement or evaluation phases when the decision-maker requires additional information or expertise validation following identification of a sensitive, inaccurate or incomplete point in the project file.

Simon writes: "The human being striving for rationality and restricted within the limits of his knowledge has developed some working procedures that partially overcome these difficulties. These procedures consist in assuming that he can isolate

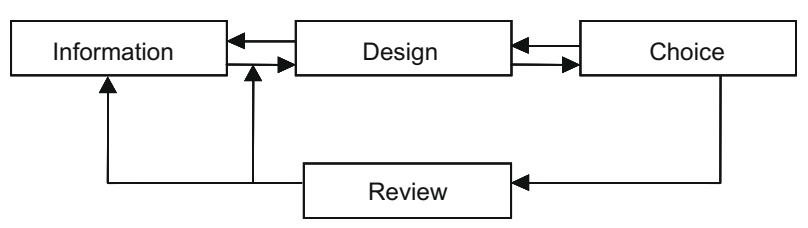

Fig. 2. Simon's IDCR cognitive model. 
from the rest of the world a closed system containing a limited number of variables and a limited range of consequences." In our framework, this bound context is modelled by a structured set of criteria that are proper to each expert in the ESCOTA's organization: the variables are the performance or rating variables related to the urgency or the priority of a maintenance operation. Then, the correctness of decisions is measured by two major criteria: (1) adequacy of achieving the desired objective; and (2) the efficiency with which the result was obtained. From our viewpoint urgency criteria refer adequacy criteria and priority criteria to efficiency criteria. ESCOTA operating domain experts of the organization seem to focus on adequacy, but the overall administrative management supervised by the service manager must pay particular attention to the efficiency with which the desired result was obtained.

Data processing, acquisition and sharing therefore lie at the heart of the software solution developed and the approach selected by ESCOTA conforms to the premise set forth in Simon's model. Our contribution lies in modelling the ESCOTA decisionmaking process for preventive maintenance in a three steps logical process with monitoring scoreboards, justification and robustness analysis of the evaluation at each level. A special attention is drawn to the multi criteria aspect of the evaluation of the NA condition, and the level of urgency and priority of the related projects.

\subsection{The measurement basis: symptom detection}

NA monitoring is conducted periodically: in-depth periodic inspections are performed every 1, 3, 5 or 6 years depending on mandatory regulatory constraints.

These inspections enable detecting deterioration symptoms or abnormalities on a given NA. Inspection reports on facility components are transmitted to field experts, who have been assigned responsibility for the various areas of intervention. With construc- tive works (bridges) for example, an evaluation method standardized by the SETRA (French Highway Engineering Agency) is used for the yearly and multiyear inspection visits via the IQOA quality imaging method [25], with the objective of synthesizing observations in order to improve maintenance service.

For instance, Fig. 3 shows the SINERGIE monitoring sheet attached to NA A808 road surface. The current routine maintenance operations, the registered abnormalities journal, the last in date inspections and the ongoing operations on NA A808 road surface can be consulted by authorized persons.

Periodic inspections are thus considered as structural condition assessments that yield a comprehensive and summary evaluation of the condition of all inspected NA. According to the IQOA imaging method for example, a partial rating that quantitatively characterizes symptom seriousness can be ascribed according to a value scale specific to the evaluation method. Thereafter, an overall rating, which summarizes the total condition of the structure and which serves to merge the various partial ratings recorded, is assigned to the structure.

The levels of seriousness quantified for the different symptoms recorded on a given NA are to be aggregated via a normalized operator, which depends on the particular facility management field: a normalized weighted average issued by the Mediterranean CETE Research Office for road surfaces, aggregation using the Max operator for the SETRA's IQOA method on engineering structures [25]. This notion of aggregation has been the topic of special development efforts.

Example 1. Let's take the example of the inspection visit of a retaining wall in an overall "fair" state of repair with a fracture on the facing. The ratings for each of the four main facility components might look like those shown in Table 1 . The (overall) IQOA rating is then $\max (1,1,2,2 E)=2 E$ with the following ordinal scale: $1<2<2 E<3<3 U$.

Example 2. The quality indices of the road surface have been defined in Table 2.

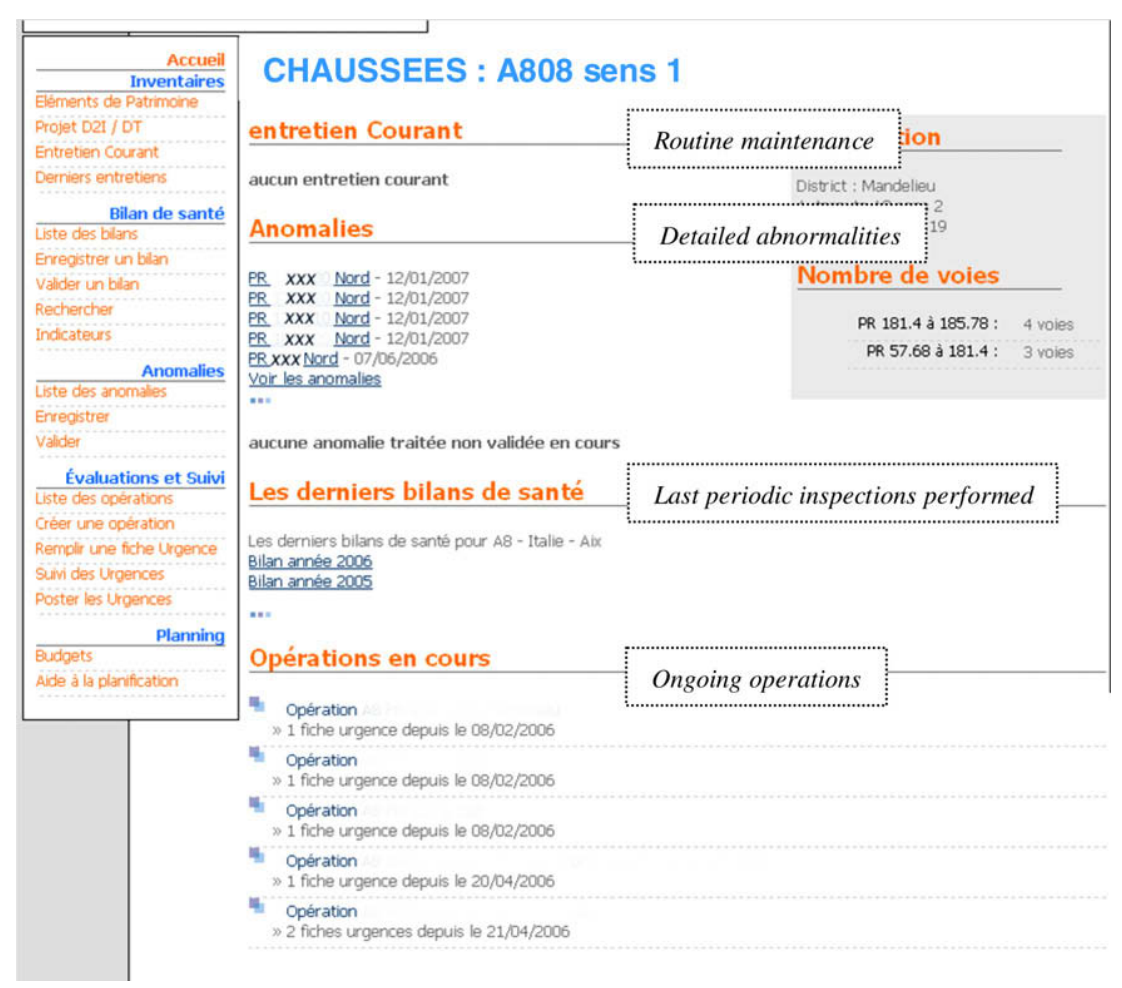

Fig. 3. Monitoring sheet for NA 
Table 1

Partial evaluation and IQOA score.

\begin{tabular}{ll}
\hline Part of network asset & Note \\
\hline Influence & 1 \\
Equipments & 1 \\
Drainage & 2 \\
Structure & $2 \mathrm{E}$ \\
NOTE IQOA & $2 \mathrm{E}$ \\
\hline
\end{tabular}

Table 2

Condition assessments for road surface.

\begin{tabular}{ll}
\hline Designation & Index \\
\hline Surface damaging index & SURF \\
Structural damaging index & STRU \\
Structural quality index & IESE \\
\hline
\end{tabular}

These indices are computed using a weighted average on a set of measurements recorded during assessments of attributes such as skid resistance, macro-texture, cracking; they then give rise to road surface condition indicators. Fig. 4 shows for highway A808 skid resistance, macro-texture and cracking measurements whose pie chart representation highlights the distribution of measures for each predefined threshold. Outside grey sectors representing the percentage of the highway where maintenance works are more recent than measures (i.e. measures then belong to the green threshold), most of the measures belong to the green or yellow threshold. Hence, A808 road surface is overall in good condition, but attention has to be paid to skid resistance considering the percentage of the orange sector. This pie chart representation enables a global vision of the NA condition. Moreover, at the top of Fig. 4, a cursor enables to compute and display the measures, thanks to the selected values of the beginning and ending kilometres posts of the NA, in order to analyse a small section of the road surface.

In brief, from a measurement-oriented perspective, once an overall rating characterizing its condition has been assigned to a NA:

- such a rating results from the aggregation of levels of seriousness quantified for the various symptoms recorded on this NA; and

- the aggregation operator applied stems from a standard imposed by an external organization, in accordance with a standard evaluation method.

For the ESCOTA motorway company, preventive structural monitoring was initiated by creating a zero point (or benchmark) on all the NA and then setting up periodic inspections [26].

The monitoring sheet in Fig. 3 lists all stored abnormalities on the A808 road surface. When travelling on the ESCOTA network, outside the inspection programs, the actors of instrumentation and inspection in their monitoring role should detect defects. Let us consider the entrance ramp on the A808 road located at kilometre posts 30 for instance where cracks are visible. The registration form in Fig. 5a is used to detail information on the localization and the lanes concerned, to select the kind of damages detected and record the inspector remarks. Once stored, the abnormality monitoring sheet (Fig. 5b) is available through the monitoring sheet (Fig. 3) enabling the expert to register his appraisal on the defects and, when necessary, to link the abnormality treatment to an existing maintenance operation. For now, there is no existing operation relative to surface repairs, but this abnormality confirms the monitoring of skid resistance assessments previously described.

\subsection{The evaluation basis: appraisal and urgency ranking}

Field experts are assigned responsibility for managing and maintaining the entire set of infrastructure. Based on these NA condition assessments, experts assess facility conditions in terms of urgency criteria for which the value scales have been established in advance (Table 3). ESCOTA has defined three possible levels of urgency for its projects, independently of the field of expertise (Table 3).

Fig. 6 provides the eight road surface expert criteria. The expert has to assess the partial urgency degree relatively to each criterion. His partial assessments depend on his interpretation of the seriousness of the damages that have been reported by inspectors and the context of the NA (location, characteristics and environment).

The urgency assessment relies on interpreting the structural condition with respect to criteria specific not only to the expert's field, but also in relative terms to the environmental characteristics, NA localization and existing abnormalities. Moreover, this assessment corresponds to a technical risk analysis and enables the expert to assign a degree of urgency to any project involving the expert's particular field.

Due to the condition assessments in Fig. 4 and to anticipate other abnormalities as in Figs. 5 and 7 shows the carriageway expert's proposal of a maintenance project concerning "ad hoc surface repairs" where the damaging description and the repair technique planned concerns most of all cracking defects. Hence, the abnormality will be linked to this operation since maintenance work takes into account cracking repairs. Following the analysis of the checking of the NA condition of the road surfaces as shown in Fig. 4, the carriageway expert identifies the road surface of A808, between kilometre posts 24 and 39, to be concerned by "ad hoc surface repairs" operation and evaluates the urgency of repairs w.r.t. this road surface ${ }^{4}$ section filling the urgency evaluation form shown in Fig. 8.

The partial ratings (Table 3 ) assigned on the basis of criteria from the field of road surfaces express the expert's interpretation of the structural condition assessment and contextual data with respect to these specialist criteria (Fig. 8). This step entails placing a technical risk analysis within an appropriate operational context. In this particular example, road surface A808 between kilometre posts 24 and 39 displays longitudinal cracking that has progressed since the last structural condition assessment. Depending on the information available over this portion of road surface (Figs. 4-6), the expert assigns an urgency rating according to each criterion and retains the possibility of justifying his intentions using natural language in an associated window (see Fig. 8). In this case, it turns out that repairing the wearing surface has become urgent since the zone involved contains curves and is heavily travelled (urban zone); safety might thus be jeopardized. The condition of the road surface is analyzed from a purely technical standpoint through the use of a surface degradation index (SURF), a structural deterioration index (STRU) and a structural quality index (IESC). This diagnostic does not alter road surface durability from its current condition (w.r.t. Fig. 5), although rapid repair work is advisable should the condition worsen. Lastly, no human health/ hygiene or social impacts are introduced (Fig. 8).

In terms of interfaces, the partial ratings recorded on the data entry form in Fig. 8 can be seen on the project evaluation data sheet generated by SINERGIE (Fig. 9). Ratings are expressed using a colour code ( $U_{1}$ red, $U_{2}$ yellow, $U_{3}$ green and 0 grey). The overall degree of urgency assigned to this project results from the

\footnotetext{
${ }^{4}$ An operation may concern several road surfaces at different points of the highway network having the same damages and needing consequently the same technical repairs.
} 
aggregation of the partial ratings assigned to the criteria listed Fig. 8: the "road surface A808 between kilometre posts 24 and 39" project is classified in level of urgency category $U_{2}$. The calculation steps used to derive this aggregated value will be discussed further below.

\subsection{The decision-making basis: strategy and priority ranking}

The urgencies $U_{i}$ relative to each project are then submitted to the validation of the infrastructure manager, who evaluates intervention priorities on the basis of priority criteria: user safety and NA security, company commitment (with respect to the State), operational constraints, etc. This manager is responsible for overseeing all fields of operation and thus plays the role of referee. The criteria are associated with standardized value scales, and three priority levels are defined for projects (see Table 4).

A project of urgency $U_{i}$ is evaluated in terms of intervention priority $P_{j}$, with this evaluation being shaped by the priority criteria, which express the perspective of the operation manager at the level of ESCOTA facility management strategy. This process involves a strategic risk analysis, i.e.: the project is evaluated according to each criterion in terms of the return on investment (ROI). The focus therefore is to appraise the risk involved in either undertaking or not undertaking the project depending on each criterion. During the evaluation of a project that is linked for example to a road surface, an assessment is made of the risk of pursuing the project or not in terms of safety, motorway operations (e.g. the potential need to close off a lane during specific time periods) or financial consequences [48]. The act of postponing a maintenance project could subsequently introduce added complexity, hence making the project more expensive.

Moreover, this level of evaluation is carried out for all sectors of specialization represented in the facility management unit: the urgencies indicated by each field expert need to be evaluated according to a unique frame of reference. The evaluations from the five experts must be harmonized; the MACBETH method [49] answers this issue and constitutes the first step of our information processing (see section 3 ). The manager then assesses and judges projects by incorporating all five facility management fields. This step serves to conclude the risk analysis taking into account the company strategy. The manager can then implement a calendar for projects that have already undergone evaluation, in order of priority, and propose multiyear schedules.

For the "road surface A808 between kilometre posts 24 and 39" example, the manager consults the urgency evaluation sheet prepared by the expert (Fig. 9). Fig. 10a shows the interface of entering partial priorities assigned by the project decision-maker for each aspect of the associated strategic risk analysis. This section of road surface is overall in good condition relative to the assessment analysis based on Fig. 4, but holes, cracks and the bad aspect produced are responsible for the $P_{2}$ partial score given to the public image criterion. Moreover, the manager knows that without a quick repair, these cracks will extend and deepen. Anticipating the repairing of cracks avoids a more expensive carriageway structure maintenance operation. After aggregating these partial evaluations, the project will ultimately be classified in $P_{2}$. Fig. $10 \mathrm{~b}$ provides the summary sheet for the project under a priority evaluation scenario (scores and comments) accessible on the manager scoreboard.

\subsection{Summary}

The three evaluation levels described above correspond to the three approaches specified in a 2005 French call for research and innovation projects, as indicated in the introduction, i.e.:
- the measurement approach for symptom detection;

- the evaluation approach for intervention urgency; and

- the decision-making approach for intervention priority.

Fig. 11 summarizes the sequencing of steps constituting the decision-making approach and reveals the new analytical aspects contributed by each hierarchical level of the decision-making process, from measurement through scheduling. The contribution and interpretation of information at each functional level enhances the decisional content of the various information flows. A decision is never made on the basis of raw information, but instead on the interpretation provided within a given context and at a given functional level.

Even if as a last resort the final decision (in this case, the decision associated to the realisation of an operation) depends on an easily identified individual, here the manager, it must still result from controlled management of the interactions taking place between all actors of the decision-making process: from the process for evaluating the structural condition of all NA to the project scheduling. The actors involved in the facility management tasks, i.e. structural inspectors, experts and supervisors, directly influence the decision based on information held and each one, depending on his/her function, responsibilities, experience and expertise, is able to offer a different interpretation. These actors cannot be dissociated from the decision-making process since they alone are able to acknowledge the diversity and complexity of the data. The SINERGIE software application is therefore aimed at encouraging information sharing and building support for the collective evaluation process.

This concludes the formal description of the decisional process for the preventive maintenance at ESCOTA's. The next section deals with the mathematical formalization of this process. It pays a particular attention to the soundness of the way assessments on the facilities conditions are achieved and processed in the computations related to the different infrastructure management activities.

\section{The MAUT-based decision aid tool SINERGIE}

\subsection{The SINERGIE technical solution}

Development of the assistance tool called SINERGIE (French acronym for Interactive Evaluation System for the Renovation and Management of ESCOTA's Infrastructure) aims at providing a significant step forward in facility management capacities at ESCOTA. ${ }^{5}$

The SINERGIE application comprises implementation of both:

- an intranet information system (IS) that handles all information available on the structures and dedicated as ESCOTA's facility management memory; and

- an interactive decision aid system designed to both support the hierarchical multi criteria evaluation process and coordinate the monitoring of facilities.

The features of this SINERGIE tool encompass:

- a knowledge database and sharing capacity since it contains not only the initial state of all network assets (NA) but also all the data on localization, traffic, urban layout;

- a risk analysis toolbox used for the evaluations of the urgency and priority of the operations. The risk analysis component entails a diagnostic for the inspectors, technical expertise for the structures expert and strategic orientations for the facilities manager;

\footnotetext{
${ }^{5}$ SINERGIE is now in its industrial phase.
} 


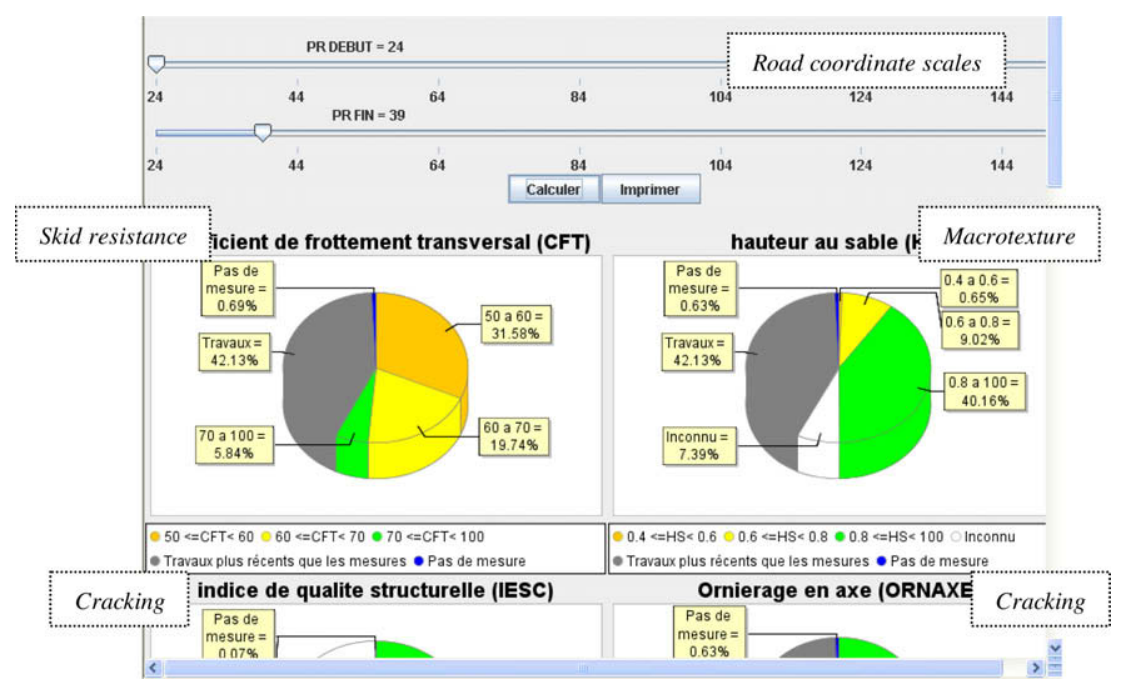

Fig. 4. Measurement for highway A808.

Table 3

Urgency levels.

\begin{tabular}{ll}
\hline Symbolic urgency level & Meaning \\
\hline$U_{1}$ & Very High urgency \\
$U_{2}$ & High urgency \\
$U_{3}$ & Moderate urgency \\
\hline
\end{tabular}

- a monitoring function for the project urgency and priority evaluations;

- an archive retrieval system that proposes a historical record of the NA condition, projects and all pertinent structural condition assessments;

- a supervision module, since the system offers a synthetic representation of any facility condition at a given point, versus time, in the form of a summary map; and

- a justification aid that enables identifying the critical dimensions of the hierarchical risk analysis that influenced the priority evaluation of a given project.

The mathematical supports of these functionalities are detailed below:

SINERGIE also offers ongoing access to information on the condition of motorway facilities. The following systems data are available for all NA components: structural condition assessments, projects completed, and projects planned (see the example of the monitoring sheet for NA on Fig. 3). Furthermore, to give a global vision of the infrastructure monitoring, a summary map allows visualizing where the thresholds used in NA structural condition assessments have been exceeded and the localization of maintenance operations planned represented with signs (Fig. 12b).

\subsection{Multi criteria evaluation and aggregation}

The two notions of rating scales (seriousness, urgency and priority) and multi criteria aggregation have already been presented in the symptoms seriousness appraisal phase, but nature of scales and aggregation operators were imposed by national civil engineering norms or safety regulations. These two key concepts within the current process, which assigns an urgency and priority to a given project, still require greater precision to be applied effectively within this hierarchical multi criteria evaluation. Indeed, no norm, no threshold, no rule is available concerning the urgency and priority appraisal phases: the assessments may suffer experts' subjectivity if no control is set up. The multi criteria evaluation process that is described in this section tackles this issue.

\subsubsection{Rating scales}

Before proceeding with the multi criteria aggregation, a step that enables calculating the urgency and priority of a given project, a few verifications need to be carried out regarding the type of the evaluation using the rating scales. This verification step proves necessary to ensure the consistency of the evaluation process. All too often in practice, aggregation techniques are employed, and typically with a weighted average, whereas only ordinal data are available for the evaluation of alternatives. For this preparatory task, the MACBETH (Measuring Attractiveness by a Categorical Based Evaluation TecHnique) method is applied [49] $]^{6}$ : in this work, MACBETH is only a first step in our multi criteria assessment process. At this point, let's offer a few minimal key notions in order to better understand the obstacles created by the evaluation scales.

Given a finite set of elements $X$, it is said that an ordinal data point is obtained whenever elements of $X$ can be sorted in decreasing order of satisfaction. Generating an ordinal data point based on the preferences of $X$ elements means that a number $n(x)$ may be associated with any element $x$ of $X$ that satisfies the following conditions:

$\forall x, y \in X:[x \mathrm{P} y \Longleftrightarrow n(x) \succ n(y)]$

and

$\forall x, y \in X:[x \mathrm{I} y \Longleftrightarrow n(x)=n(y)]$

where the relation $P$ ("is more attractive than") is asymmetric, while the relation $I$ ("is as attractive as") is an equivalence relation. One example for $n(x)$ is to take the number of $X$ elements lying after $x . n(x)$ then generates an ordinal scale. It can be noted that this procedure does not allow a unique definition of $n(x)$ values.

On the basis of this ordinal information, it is feasible to build an interval scale by collecting, in the form of differences, the preference intensities between $X$ elements:

$n(x)-n(y)=k \alpha, k \in \mathrm{N}$

\footnotetext{
${ }^{6}$ We do not propose new advances regarding the MACBETH method in this paper (we have considered the extension of MACBETH to Choquet integrals in other works $[33,50])$
} 


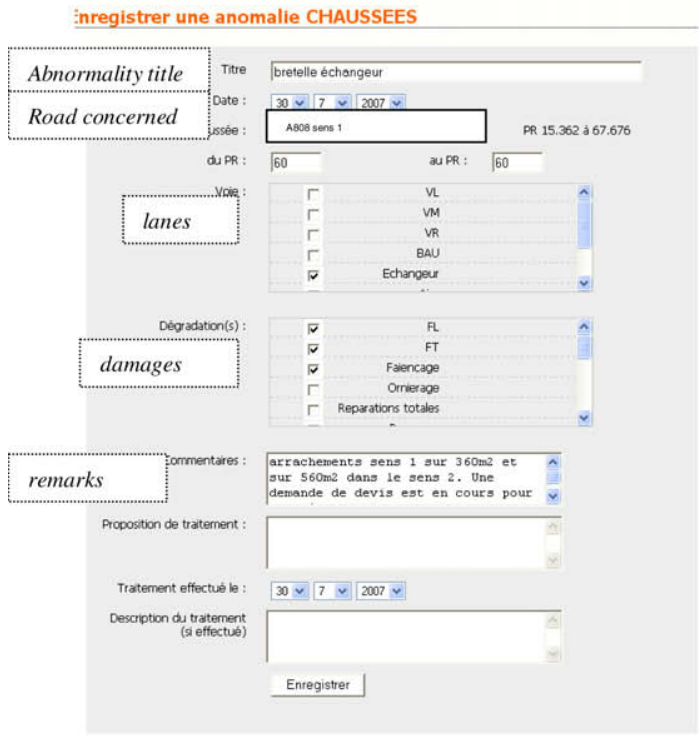

5a) Abnormality registration form

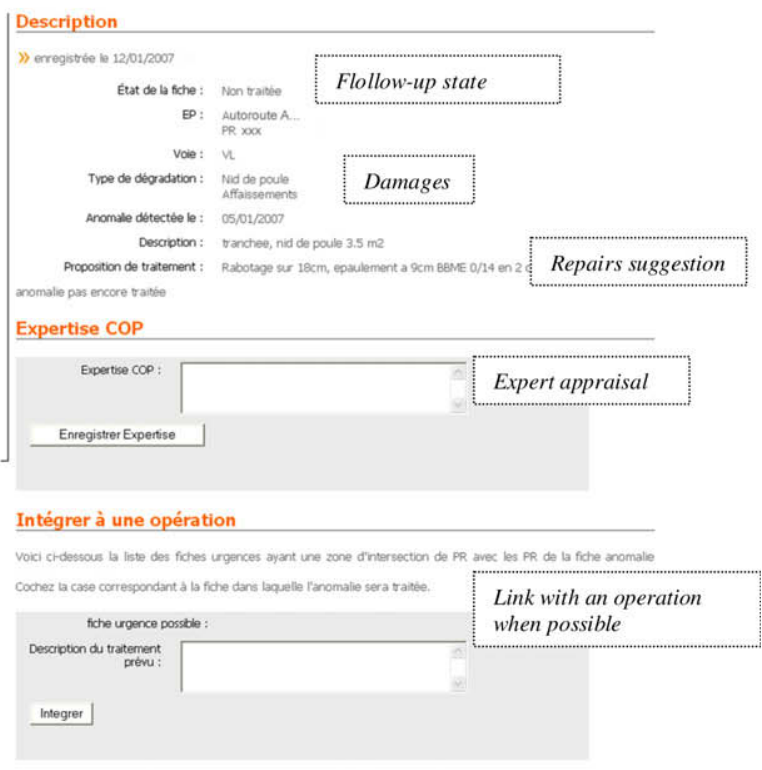

5b) Abnormality monitoring sheet

Fig. 5. Abnormality sheet.

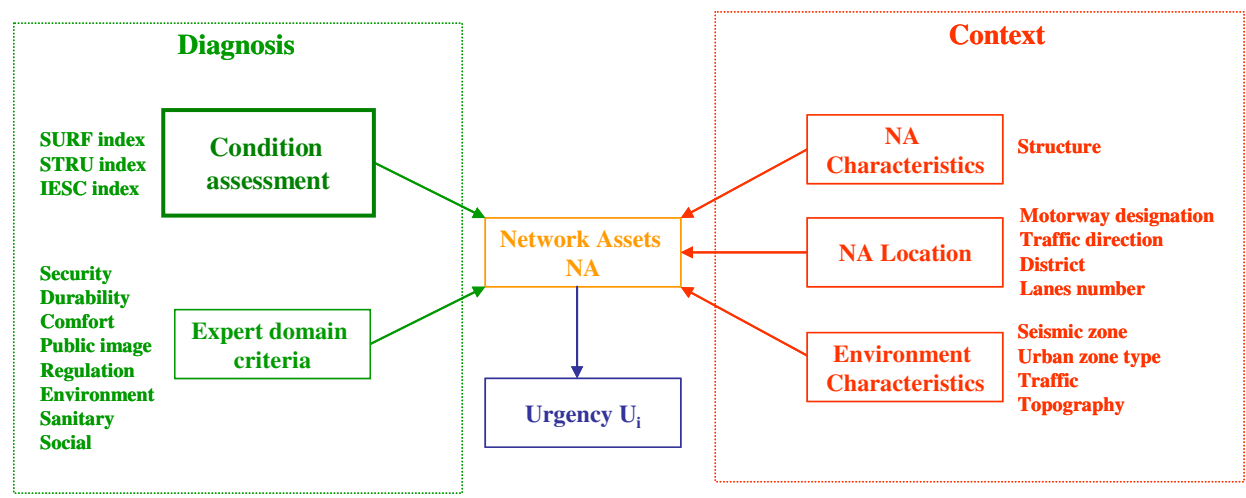

Fig. 6. Urgency criteria for road surface.

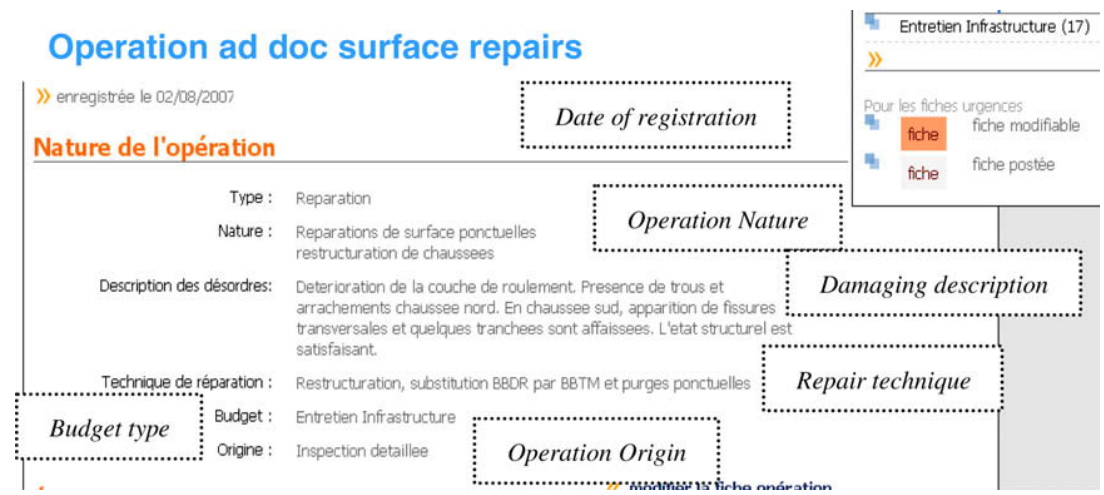

Fig. 7. Maintenance project example.

where $k$ characterizes the preference intensity and $\alpha$ is introduced to respect the domain bounds (e.g. $[0,1])$. The interval scale is obtained by solving a system of equations of the type (1)-(3), supplemented by a comparison of expressions at the extreme values 0 and 1 over the domain [0,1]: $1-n(x)=k \alpha\left(3^{\prime}\right)$ and $n(x)-0=\mathrm{k} \alpha\left(3^{\prime \prime}\right)$.
The MACBETH method is used to create an interval scale that quantifies the value judgments w.r.t. one criterion an individual could have concerning the elements of a finite set. In this instance, the focus lies in verifying that both the experts and operations manager are indeed employing interval scales for their urgency and priority ratings. MACBETH proposes a surveying mode based 
on opinions expressed regarding the potential difference in attractiveness between any two elements of $X$. This same principle also lies at the origin of the name MACBETH. In this application, the MACBETH method is a preliminary step that guides the experts and operations manager in building their cardinal evaluation scale.

As an example, the expert assigned responsibility for the "Road surfaces" field will be asked about the intensity difference existing, in terms of urgency with respect to the safety criterion, between two maintenance projects specific to two road surface segments. This procedure is carried out on a set of projects compared two at a time (i.e. a learning basis) in order to identify the scale being used by the expert. The more maintenance projects are compared by the expert w.r.t. safety criterion, the more equations of type (2) and the more precise the interval scale. The expert must at least provide two maintenance projects that correspond to the lowest and the highest ratings, then a linear interpolation is assumed between the extreme values. Fig. 13 illustrates this process for criterion Safety. The "Road surfaces" field expert compares 10 projects $\{$ A. . J $\}$ two at a time. $\{$ A. . J $\}$ is the learning basis of projects to identify the Safety scale. The real names of projects are not given for confidentiality reasons. Two fictive projects urgent (highly urgent) and peu_urgent (not urgent at all) complete the training base (the extreme values). The "positive" label in Fig. 13 introduces a more flexible constraint because it simply replaces any label with a higher degree than weak. The elements of the comparison matrix express the expert ranking and provide the required set of constraints of types (3). Solving this set of equations gives the Safety scale, i.e. the 10 projects are distributed over the interval $[0,100]$ consistently with the set of constraints: the resulting cardinal scale is given at the right side of Fig. 13.

Note that deriving cardinal information on the preference intensities of criteria must be consistent with the selected aggregation method. The "classical" MACBETH method guarantees this consistency when the aggregation is a weighted average; however extensions of the method have been proposed [50].

\subsubsection{Multi criteria aggregation}

One of the problems associated with multi criteria decision aid is to be able to sort the solutions (in this case, projects in terms of urgency and priority) by taking all criteria into account [51]. Besides the aggregation on symptom indicators, where the aggregation operator has been imposed by institutional partners (the Max operator for the IQOA campaign on bridges, weighted average according to the CETE protocol for road surfaces), the expert aggregation operators still need to be defined to calculate the overall degree of urgency associated with a given project, in addition to the service manager aggregation operators to evaluate the overall degree of project priority. Many references deal with the identification of the $h$ operator [52,53].

With the cardinal approach, the focus lies in building a function $h:[0,1]^{n} \rightarrow[0,1]$, such that for each project $O P^{k}$ described by its partial evaluations: $\left(u_{1}^{k}, \ldots, u_{n}^{k}\right): h\left(O P^{k}\right)=h\left(u_{1}^{k}, \ldots, u_{n}^{k}\right)$, where $h\left(O P^{k}\right)$ represents the overall evaluation of project $O P^{k}$ relative to the $n$ criteria and where $u_{i}^{k}$ is the partial evaluation of $O P^{k}$ with respect to (w.r.t.) criterion $i$.

This function $h$ is then referred to as the operator. Various operators allow expressing the simultaneous fulfilment of objectives (decision-maker's conjunction attitude, e.g. $h\left(u_{1}, u_{2}, \ldots, u_{n}\right)=$ $\prod_{i=1}^{n}\left(u_{i}\right)$ ), redundancy (disjunction attitude, e.g. $h\left(u_{1}, u_{2}, \ldots, u_{n}\right)=$ $1-\prod_{i=1}^{n}\left(1-u_{i}\right)$ ), and compromise (included among these operators would be arithmetic, geometric or harmonic averages, symmetric sums).

The identification of the $h$ operator can be broken down into two stages. Decision-maker behaviour can be determined through requesting a verbal assessment of the level of compatibility between consequences and objectives for a limited set of actions, which then makes it possible to quickly designate the type of aggregation to apply: conjunction, compromise or disjunction. Once one of these three basic decision-maker attitudes has been ascribed, it becomes possible to proceed with using families of parameterized functions in order to refine the aggregation operator determination step: the choice of $h$ then becomes a parametric identification problem [54]. In the operators presented above however, the criteria play a symmetric role. In many of these practical cases, it may prove necessary to introduce the notion of relative importance of criteria (RIC).

The notion of RIC naturally leads to introducing weighting distributions on criteria within the scope of the formalism provided above by generalizing operators if and where possible.

The most typical case associated with such operators is the weighted average:

$\forall\left(u_{1}, u_{2}, \ldots, u_{n}\right) \in[0,1]^{p}, \quad h\left(u_{1}, u_{2}, \ldots, u_{n}\right)=\sum_{i=1}^{n} p_{i} u_{i}^{k}$

The notion of weighting may be transposed onto many aggregation operators, once the project displays an underlying additive structure [17]. As such, when $h$ is of the form $\psi^{-1}(\psi()+.\psi()$.$) , an exten-$ sion to the previous equation can be proposed, i.e.:

$\left.\bar{h}\left(u_{1}, u_{2}, \ldots, u_{n}\right)=\Psi^{-1} n \sum_{i=1}^{n} p_{i} . \Psi\left(u_{i}\right)\right) \quad$ where $\sum_{i=1}^{n} p_{i}=1$

As an example, for the product, when setting $\psi=\mathrm{Log}$, the following result is obtained: $\bar{h}\left(u_{1}, u_{2}, \ldots, u_{n}\right)=\prod_{i=1}^{n}\left(u_{i}\right)^{n p_{i}}$.

In this application, each step of a project evaluation (i.e. levels of urgency and priority) corresponds to the criteria relative to each actor's (experts' and manager's) field of specialization (see Figs. 8 and 10). The evaluation at a given level thereby consists in estimating the project benefit with respect to the entire of set of analysis criteria associated with the decision-maker's function: a partial score is assigned to each one of these criteria, with all scores then being circulated up the decision-making hierarchy via aggregation operators that serve to assign an overall score to the project in terms of urgency and priority. The notion of relative importance of criteria proves critical to this particular application: it enables distinguishing the role of each criterion relative to the marginal contribution in developing overall evaluations.

Since our goal is more oriented to presenting a methodology than to implementing sophisticated mathematical tools that would interfere with the explanation, ${ }^{7}$ it is considered that the strategies of each expert and the manager only contain the following characteristics:

- the relative importance of criteria is to be taken into consideration;

- compensation between partial scores is authorized;

- monotony is strict $\left(u_{i}>u_{i}^{\prime} \Rightarrow h\left(u_{1}, \ldots, u_{i}, \ldots, u_{n}\right)>h\left(u_{1}, \ldots\right.\right.$, $\left.u_{i}^{\prime}, \ldots, u_{n}\right)$ ); and

- criteria are independent.

The weighted average mean (WAM) is an operator that incorporates these characteristics. It will therefore be considered that the decision-making strategies, associated with the experts or manager, are simple weighted averages.

The weighted average type of aggregation is able to model the RIC through standardized (weighting) coefficients, to be interpreted as substitution rates between criteria:

\footnotetext{
${ }^{7}$ More complex operators specifically devoted to the modeling of the interactions between criteria were employed for equivalent purposes in $[33,34]$.
} 


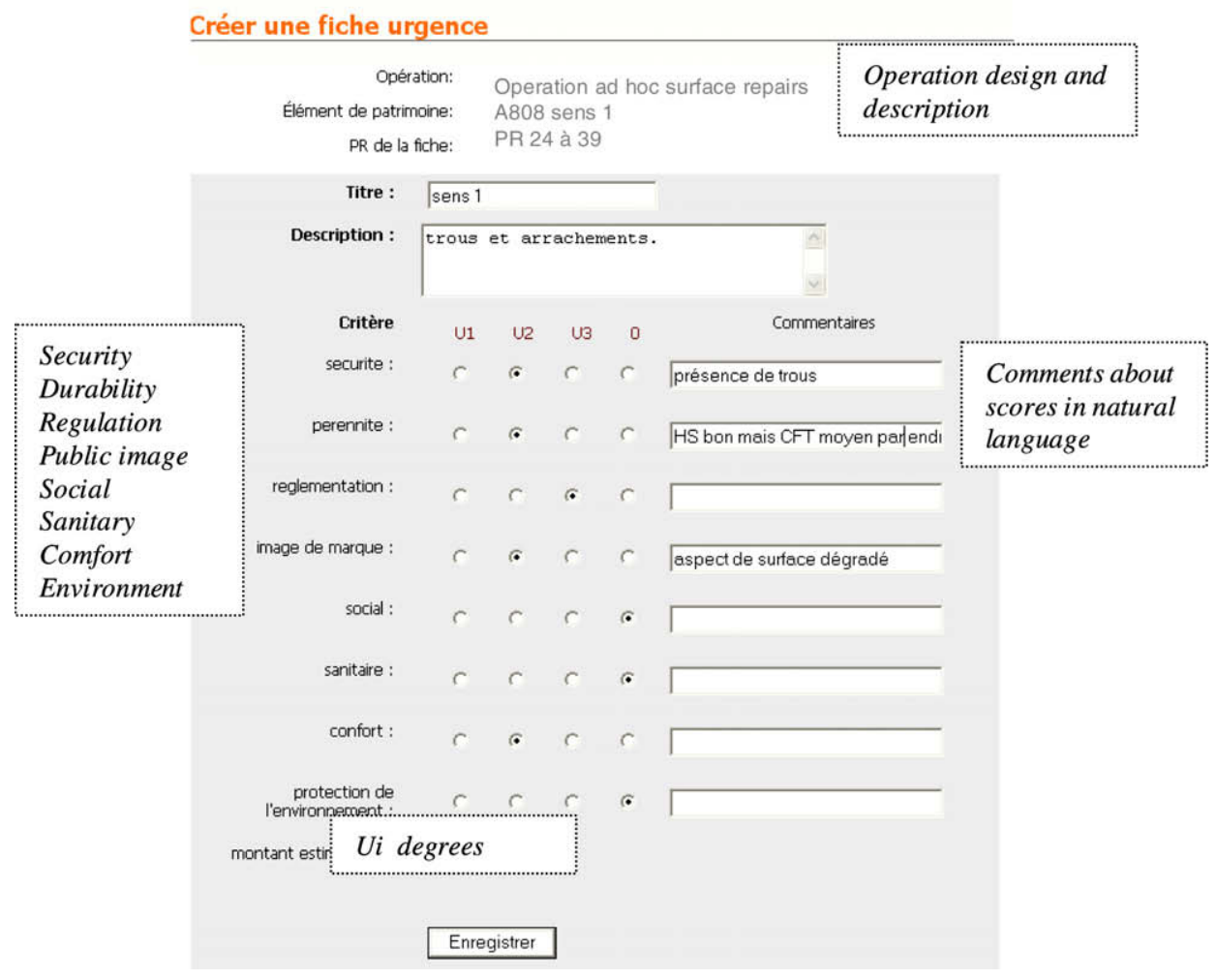

Fig. 8. Urgency evaluation form example.

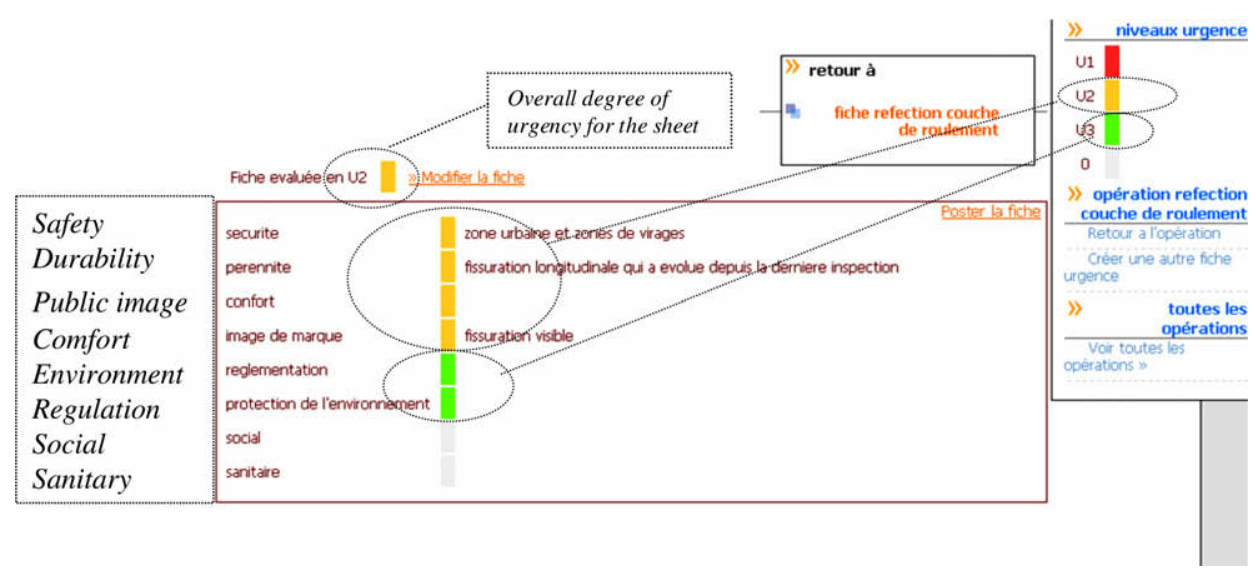

Fig. 9. $U_{2}$ urgency project evaluation data sheet.

Table 4

Priority levels.

\begin{tabular}{ll}
\hline Symbolic priority level & Meaning \\
\hline$P_{1}$ & Very high priority \\
$P_{2}$ & High priority \\
$P_{3}$ & Moderate priority \\
\hline
\end{tabular}

$h_{w}\left(O P^{k}\right)=h_{w}\left(u_{1}^{k}, u_{2}^{k}, \ldots, u_{n}^{k}\right)=\sum_{i=1}^{n} w_{i} u_{i}^{k}$,

where the $u_{i}^{k}$ values are the scores assigned to a given project $O P^{k}$ vs. each one of the $n$ criteria associated with an ESCOTA decisionmaking function (whether experts or manager) and $\mathrm{w}_{\mathrm{i}}$ the weights associated with criterion $i$ for this function. The term $w_{i} u_{i}^{k}$ represents the marginal contribution of criterion $i$ to the evaluation of $\mathrm{OP}^{\mathrm{k}}$. This concept will play a key role in the justification functionality, as will be shown below.
Several methods exist to identify and perform aggregation process with a WAM. The Analytic Hierarchical Process, AHP, is probably the most famous one in industry [28]. However, because we have already used the MACBETH method for the scales identification, we chose to keep it for the WAM parameters identification step. The technical reasons of this choice are summarized in the next paragraph.

MACBETH explicitly guarantees the consistency between the commensurable scales it aggregates and the WAM operator it identifies (the scales identification is part of the method). The weight scale is in fact an interval scale itself that can be obtained by applying the procedure described in Section 3.2.1. AHP or MACBETH work with indirect information to identify the weights of a WAM: pair wise comparisons are done considering the ratio or difference of attractiveness rather than attractiveness itself (the set of comparisons enables to identify the weight scale). These indirect methods for weight identification are rather well received in 


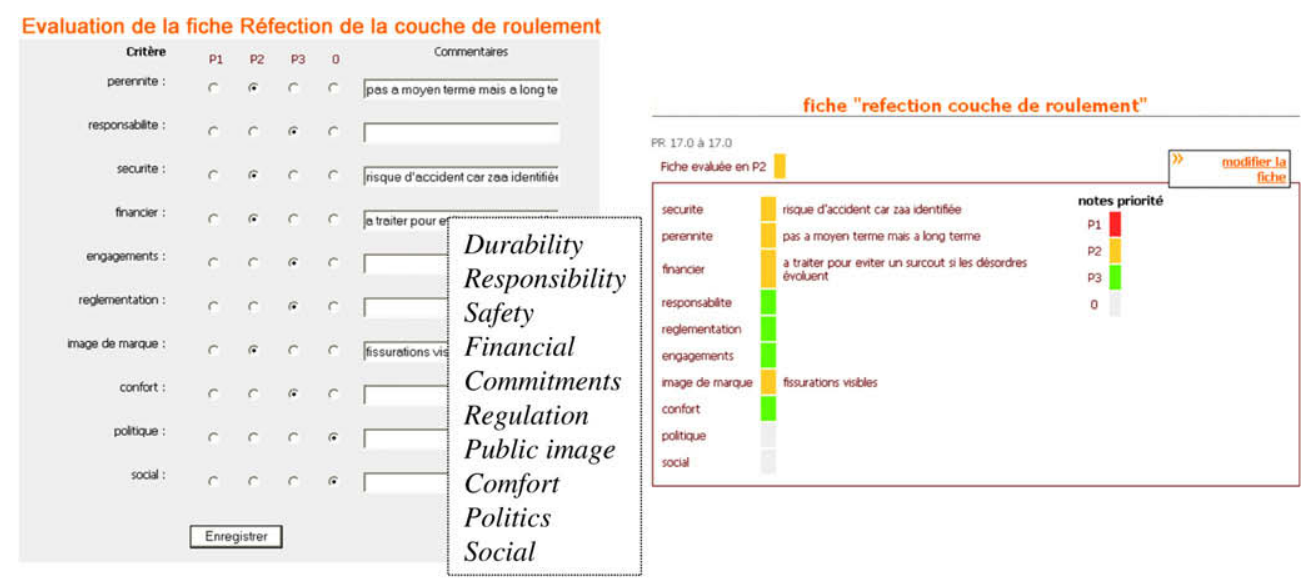

10a) Priority evaluation form

10b) Priority project evaluation data sheet

Fig. 10. Project priority evaluation.

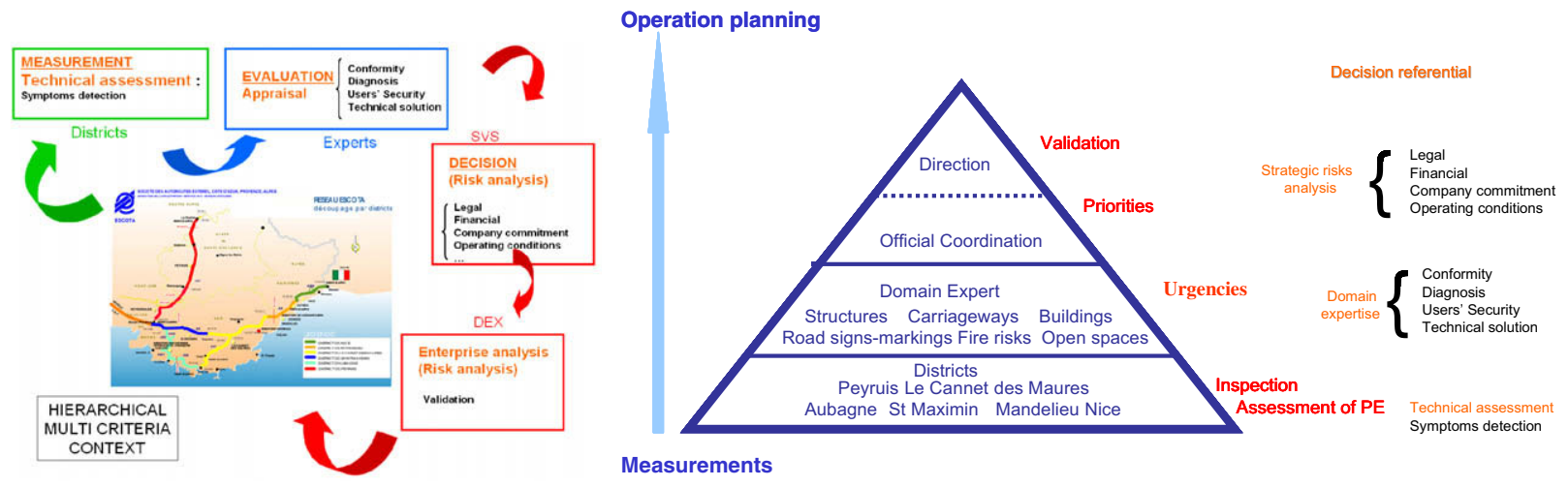

Fig. 11. A hierarchical multi criteria context.

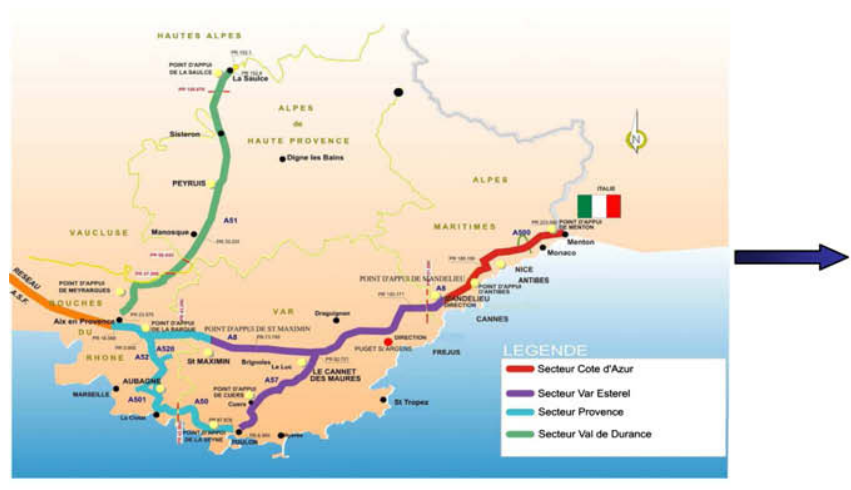

12a: ESCOTA network

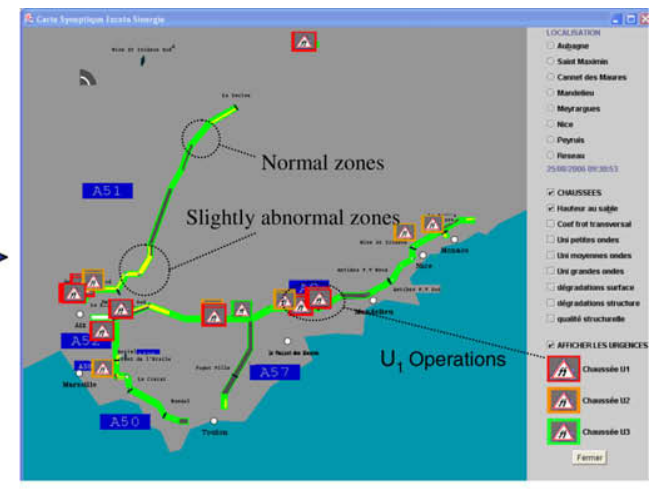

12b: Network structural assessments synoptic

Fig. 12. Cannet des Maures supervision synoptic map.

industry because they explicitly highlight the role of the experience and the know-how of the company's experts.

A point in favour of Macbeth is that it provides a complete information processing that deals with our issue (our problem is not only to identify the adequate WAM operators but also to support experts in designing the scales of the criteria to be aggregated) and that is consistent with the measurement theory [55]. MACBETH proposes the same basic principle to consistently identify the interval scales and the WAM operator (the weight scale is an interval scale that can be determined following Section 3.2.1);
AHP assumes the aggregated scales to be the ratio scales that have been identified elsewhere. Let us give some explanations about this comment. An aggregation operator must be meaningful with regard to the scales of the alternative scores it aggregates. In MAC$\mathrm{BETH}$, criteria scales are interval scales that are consistent with a WAM operator: any affine transformation of an interval scale does not affect the WAM alternatives ranking. Let us consider an example. Imagine you try to control the heating of a room in spring. This control depends on the inside temperature $\left(T_{R}^{C^{\circ}}\right)$ in the room and the outdoor temperature $\left(T_{\text {Out }}^{C_{0}}\right)$. Suppose temperatures are given 

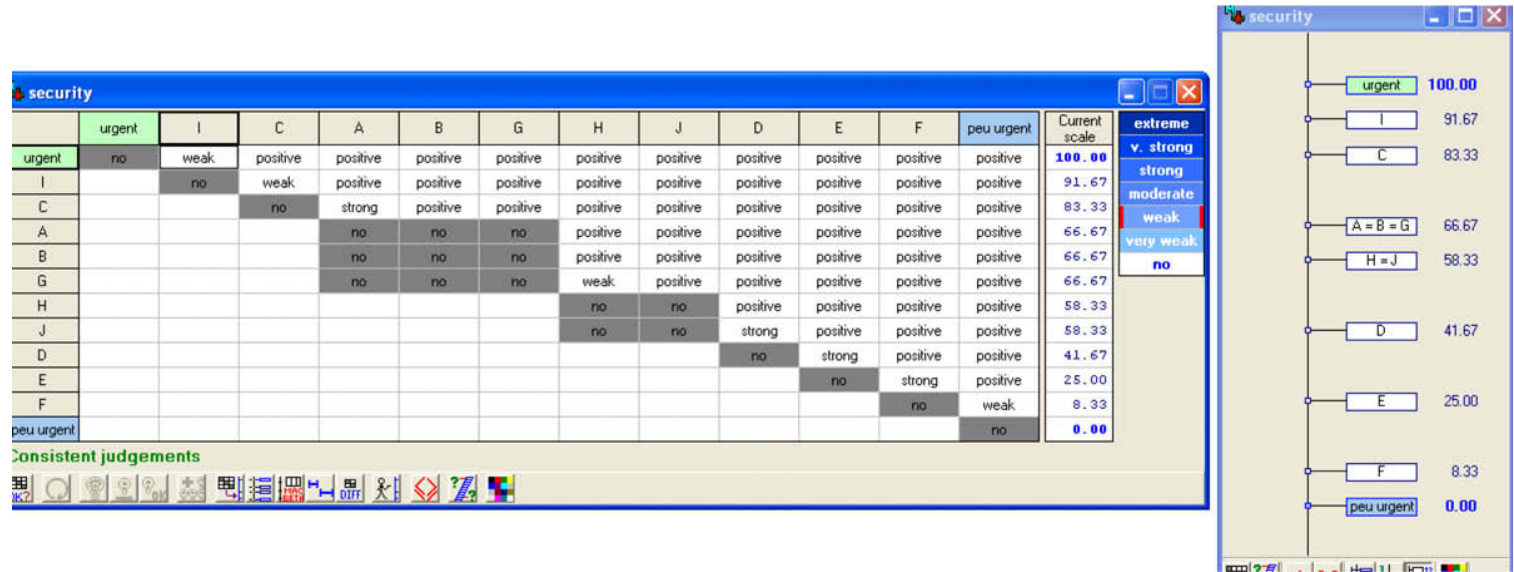

Fig. 13. MACBETH - two at a time comparison of projects and cardinal scale for Safety criterion.

in Celsius degrees and imagine the simple control rule "if $T_{R}^{C^{\circ}}-T_{\text {Out }}^{\mathrm{C}^{\circ}}$ is below 5 degrees then start heating". For example, when $T_{R}^{C^{\circ}}=22 C^{\circ}$ and $T_{\text {Out }}^{C^{\circ}}=15 C^{\circ}$ then you start heating. Now imagine the temperature unit is changed. You convert Celsius in Kelvin: $T^{K}=T^{C^{\circ}}+273$ (affine transformation). The heating rule remains the same and $T_{R}^{K}-T_{\text {Out }}^{K}=295-288>5$. The difference operator used in the control rule is consistent with the temperature interval scales. Now let us consider the following control rule "if $T_{R}^{\mathrm{C}^{\circ}} / T_{\text {Out }}^{\mathrm{C}^{\circ}}$ is below 1.2 then start heating". The difference has been replaced by the temperature ratio in the control rule. Then, if you change the temperature unit as proposed above, you can start heating or not depending on the unit temperatures measures: the division (ratio) is not meaningful with the temperature interval scales; the Celsius-Kelvin transformation can entail abnormal behaviours. Namely, $T_{R}^{C^{\circ}} / T_{\text {Out }}^{C^{\circ}}=1.46>1.2$ but $T_{R}^{K} / T_{\text {Out }}^{K}=1.02<1.2$.

The only idea beyond this remark, which is of importance for our work, is that MACBETH supports the whole data processing that ensures that measurement scales and aggregation operator are consistent (the weights of the WAM define an interval scale). The first step in our evaluation process is thus to check that experts preferences can be modelled with a WAM operator but also that their evaluation scales are interval ones, consistent with a WAM aggregation.

Let us consider our example again. MACBETH is applied to identify the weights of the WAM operator for the "Road surface" expert. The weight scale is a particular interval scale (namely, a weight is a mere particular value of the WAM operator: $\operatorname{WAM}\left(0, \ldots, 0,1_{i}\right.$, $\left.0, \ldots, 0)=w_{i}\right)$. The two at a time comparison is carried out over the 8 urgency criteria of the road surfaces field expert (Fig. 8). The resulting interval scale of weights is given in Fig. 14. At this stage of the modelling, the carriageway expert has identified his 8 urgency scales and his WAM parameters. He is now supposed to be able to compute the global degree of urgency of any project when partial quotations are available, w.r.t. each criterion.

For the experts, criteria weights are preliminarily defined and then remain identical through all evaluations; they depend on the targeted field of intervention and the expert's background. The manager evaluates the projects in all fields using the same priority criteria, yet these criteria are not ascribed the same importance depending on the field under consideration. On the other hand, the priority criteria weights are defined for each individual

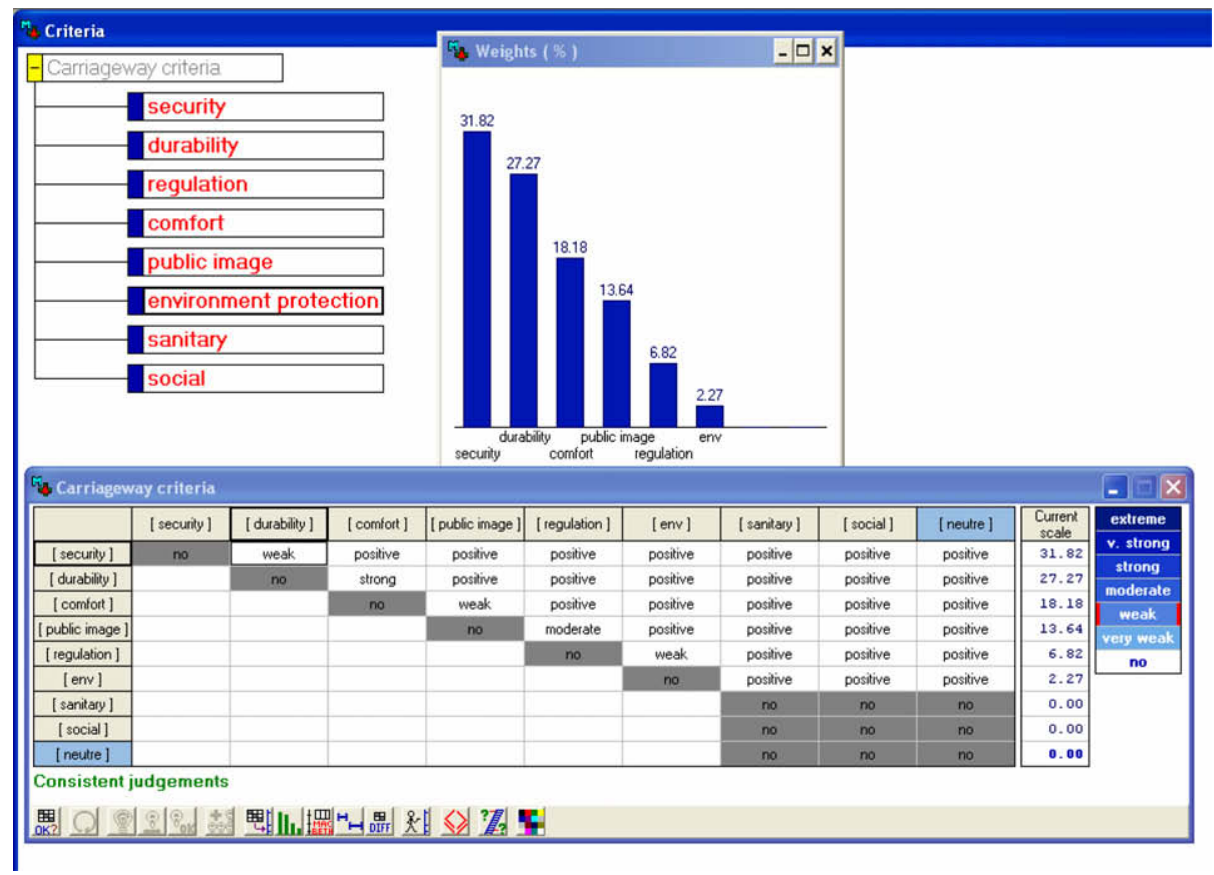

Fig. 14. MACBETH - two at a time comparison of road surface field expert criteria and weights identification. 
facility management field by the manager. This procedure guarantees priority evaluation compatibility from one field to the next.

\subsubsection{Discrete cardinal scales of urgency}

The previous subsections merely aimed at justifying the choice of MACBETH as the mere initial step of our multi criteria assessment process. However, other problems were raised by the application. Partial scores aggregation does not cause any further problems when quotations referred to continuous cardinal scales as previously explained. It is more questionable when partial scores are expressed on a discrete or finite scale. Indeed, ESCOTA experts express their assessment w.r.t. each criterion on a finite set of 3 labels $\left\{U_{1}, U_{2}, U_{3}\right\}$. The different $U_{i}$ define a discrete cardinal scale. However, computing the WAM value necessitates assigning numerical values to each $U_{i}$. In the following, we describe the way this assignment can be achieved in a consistent manner with previous MACBETH identification phases.

Although experts are used to deal with aggregation procedure (IQOA, CETE Method, etc.), the way they give their assessment in natural language raises another problem [56]. These labels are commonly converted into numerical values to perform the aggregation process. No particular attention is generally paid to this "translation". However the consequences over the aggregation results are damageable. In civil engineering, the culture of numbers is strongly developed. People commonly manipulate symbolic labels but may convert them into more or less arbitrary numerical values when necessary without the necessary care. This cultural viewpoint explains why an aggregation operator is generally preferred to a rule base whereas appraisals are expressed in terms of symbolic labels [56]. A completely symbolic evaluation over finite scales could be envisaged [57]. We have implemented the ideas introduced in both [56,57] for a purely symbolic treatment of evaluations. Some interesting results about the non linear behaviour of the symbolic WAM have been pointed out in [58]. These results are not presented here for paper length reasons, but also for practical reasons: the symbolic approach required a huge learning data base of projects and implied non common mathematical concepts for ESCOTA engineers [58].

Let us illustrate the scales problem with the following example. Let us suppose that the semantic universe of an expert w.r.t. the seriousness of a symptom is: \{insignificant, serious, alarming\}. We can imagine that a corresponding possible set of discrete numerical values (in $[0,1]$ ) could be: $\{0 ; 0.5 ; 1\}$. There are several assumptions behind this translation concerning the nature of the scale. Let us just note here that the numerical values are chosen equidistant. Now let us consider another semantic universe: \{insignificant, minor, alarming\}. This time, the associated set of numerical values $\{0 ; 0.5 ; 1\}$ intuitively appears more questionable. The expert should prefer $\{0 ; 0.25 ; 1\}$. When seriousness degrees of several symptoms are to be aggregated, the result of the WAM aggregation strongly depends on the choice of the set of numerical values. Furthermore, in any case, the numerical WAM value does not necessary belong to $\{0 ; 0.5 ; 1\}$ or $\{0$; $0.25 ; 1\}$. It must then be converted into the convenient label in return. The way labels are converted into numerical values (and back) coupled to the commensurability of the scales of the dimensions to be aggregated can lead to serious problems when aggregation is performed without any care. In this section, we propose the practical methodology that was implemented to build finite partial valuation scales, consistent with the WAM aggregation.

Let us still consider the expert assigned responsibility for the "Road surfaces" field. A continuous cardinal scale has been identified with the MACBETH method for the urgency scale of each criterion by using a limited learning base of 23 projects (Fig. 15). We know in practice that the expert's scores only take four values: 0 and $\left\{U_{1}, U_{2}, U_{3}\right\}$ (the $U_{i}$ are associated to a colour code in Fig. 15). The problem is now to assign a set of numerical values $\left\{u_{1}^{i}, u_{2}^{i}, u_{3}^{i}\right\}$ to $\left\{U_{1}, U_{2}, U_{3}\right\}$ for criterion $i$. Let us suppose the continuous cardinal scale for criterion $i$ has been identified with a training set of $q$ projects as explained in 3.2.1. ( $q=23$ in this example). These projects are grouped into 3 clusters corresponding to $U_{1}, U_{2}$, $U_{3}$. The computation of the clusters and their associated centres is achieved by minimizing the quadratic difference $\sum_{k=1}^{3} \sum_{j=1}^{q_{k}}\left(u_{k}^{i}-\right.$ $\left.u^{i}\left(O P_{j}\right)\right)^{2}$ where $q_{k}$ is the number of projects in class $U_{k}$ $\left(\sum_{k=1}^{3} q_{k}=q\right)$ and $u^{i}\left(\mathrm{OP}_{\mathrm{j}}\right), j=1 . . \mathrm{q}$, the urgency degree of a project $O P_{j}$ w.r.t. criterion $i$ computed with Eq. (6) issued from the MACBETH step.

In the example of Fig. 13, the computation of clusters gives: $u_{1}^{\text {sec urity }}=0.91, u_{2}^{\text {sec urity }}=0.52$ and $u_{3}^{\text {sec urity }}=0.11$. This assignment is repeated for each criterion relative to the road surface field. Then, the WAM can be numerically computed:

- For each criterion $\mathrm{i}, i=1 . . n(n=8)$, a value $U_{k}$ is affected to a project $O P$. Let us note this urgency degree $U_{k(i)}$;

- $O P$ is thus described by its vector of urgency degrees

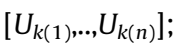

- The corresponding vector of numerical values is: $\left\{u_{k(1)}^{1}\right.$, $\left.u_{k(2)}^{2}, \ldots, u_{k(n)}^{n}\right\}$.

Then,

$W A M(O P)=\sum_{i=1}^{n} p_{i} \cdot u_{k(i)}^{i}$

The last constraint to be satisfied is that the WAM values must be converted in return into the semantic universe $\left\{U_{1}, U_{2}, U_{3}\right\}$. The output of the WAM operator must be discretized in $\left\{U_{1}, U_{2}, U_{3}\right\}$. The problem is thus to determine the centres of the $U_{k}$ clusters of the aggregated urgency scale (WAM values).

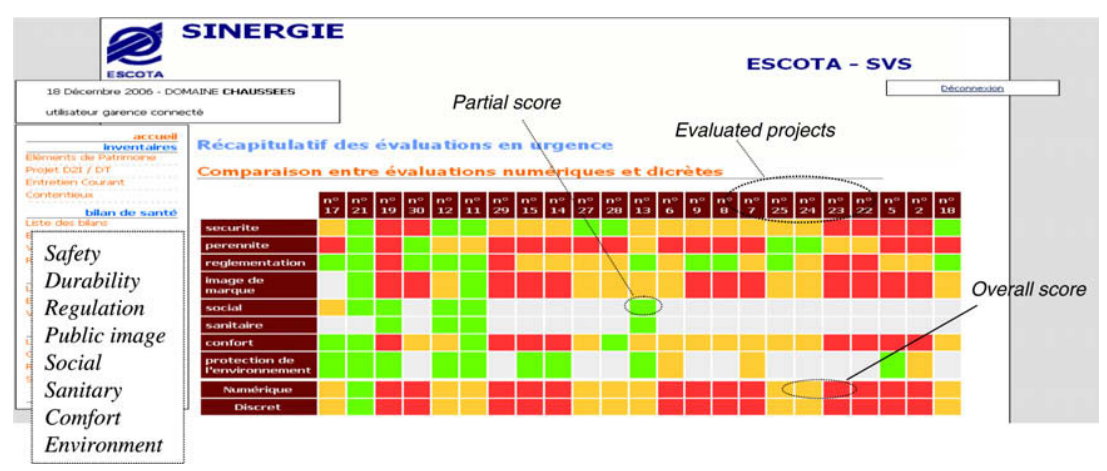

Fig. 15. Tests on the evaluation method over a base of 23 projects: $\square_{u_{1}} \mathrm{U} 1, \square_{\mathrm{u}} \mathrm{U} 2$, $\square_{\mathrm{u}} \mathrm{U} 3$. 
Let us note that the WAM operator is idempotent. Therefore, we must have:

$\forall U_{k}, k \in\{1,2,3\}, \operatorname{WAM}\left(U_{k}, \ldots, U_{k}\right)=U_{k}$

A sufficient condition for (8) is that the centres of the $U_{k}$ clusters of the aggregated urgency scale are the images of the corresponding $U_{k}$ centres of the partial urgency scales by the WAM function, i.e.:

$W A M\left(u_{k}^{1}, . ., u_{k}^{n}\right)=\sum_{i=1}^{n} p_{i} \cdot u_{k}^{i}=u_{k}^{A g}$

where $u_{k}^{A g}$ is the centre of class $U_{k}$ in the aggregated urgency scale.

Consequently, when a project is defined by its partial urgency vector $\left[U_{k(1)}, ., U_{k(n)}\right]$, Eq. (4) provides the numerical value.

$W A M(O P)=\sum_{i=1}^{8} p_{i} \cdot u_{k(i)}^{i}$

Then, the attribution of a class $U_{k}$ in the aggregated urgency scale is obtained through the following calculation:

$\operatorname{Arg} \min _{k}\left|u_{k}^{A g}-\sum_{i=1}^{n} p_{i} \cdot u_{k(i)}^{i}\right|$

The value of $k$ in $\{1,2,3\}$ that minimizes the expression in (11) provides the class $U_{k}$ of project $O P$.

Fig. 16 summarizes the whole evaluation process of a project $O P$. The validation of this process has been carried out with a test base of 23 projects in the road surface field. The expert has analyzed each of these projects. For each of them, he has attributed urgency degrees in the ESCOTA normalized semantic universe $\left\{U_{1}, U_{2}, U_{3}\right\}$ w.r.t. every of his 8 criteria. Then, the aggregated urgency degree in this semantic universe can be computed using the 3-step process described in this paper (white arrows in Fig. 16). Besides these computations, the expert has been asked to directly attribute an overall urgency degree to each of the 23 projects (grey arrow in Fig. 16).

Fig. 15 reports these data. The last line corresponds to the direct expert evaluation (grey arrow in Fig. 16). The last but one line provides the corresponding computed values with the 3-step method (white arrows in Fig. 16). No error has been observed. However, the poor semantic universe - only 3 labels - implied in our application can also partly explain such a perfect matching.

\subsubsection{Project urgency and priority evaluations: Summary}

Let's now consider a project OP that implies a set of NA $\left\{E P_{1}, \ldots\right.$, $\left.E P_{n}\right\}$, for which the corresponding evaluation process is presented

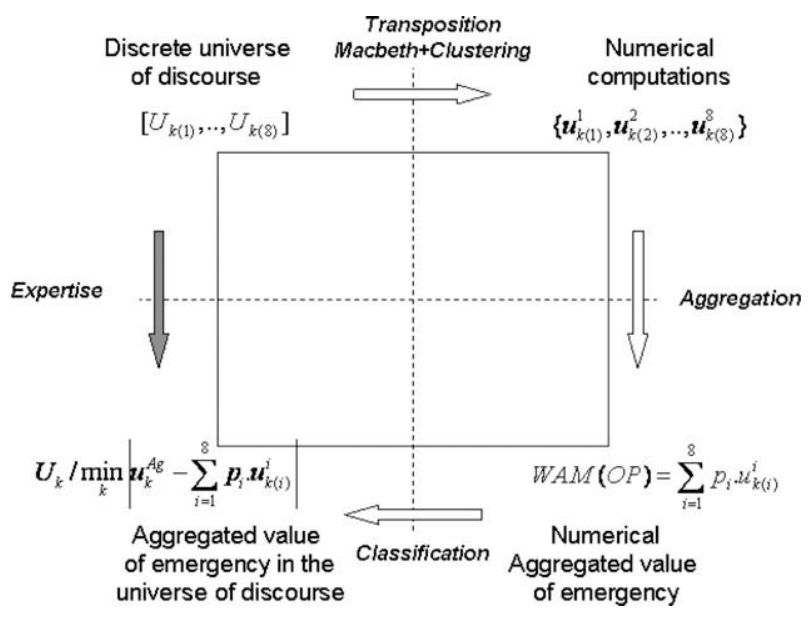

Fig. 16. Evaluation process of a project. in Fig. 16. It will be assumed that the context is set within one of the five facility management fields.

3.2.4.1. Intervention urgency evaluation. Based on the structural condition assessments and/or inspector requests, the expert responsible for the particular field evaluates each project NA and relates a score that depends on each urgency evaluation criterion. This score may eventually be enriched by a comment intended to explain the reasons of the score assignment. Each partial score is expressed in the value space $\left\{U_{1}, U_{2}, U_{3}\right\}$. The weighted average of scores using the set of criteria weights established by the expert also concentrates its values in this finite space for each NA. Three groups of NA are thus obtained for the project: $\left\{U_{1}\right\} O P,\left\{U_{2}\right\} O P$, and $\left\{U_{3}\right\} O P$.

3.2.4.2. Intervention priority evaluation. For each NA placed in the $U_{1}$ category, the manager associates a partial score expressed in the value space $\left\{P_{1}, P_{2}, P_{3}\right\}$, ultimately enriched by a comment for each priority criterion in order to substantiate the score assignment. The weighted average of scores using criteria weights defined by the manager also finds its values in this space $\left\{P_{1}, P_{2}\right.$, $P_{3}$ \} (Fig. 17). The manager proceeds similarly for the $U_{2}$ and $U_{3}$ categories of NA, which results in three NA groups categorized as $P_{1}$, $P_{2}$ or $P_{3}$.

The potential of having three NA categories, respectively, placed in $U_{1}, U_{2}$ and $U_{3}$ that get evaluated respectively in $P_{1}, P_{1}$, and $P_{2}$ needs to be underscored. The urgency and priority scales are in fact independent since they do not pertain to the same set of concerns.

3.2.4.3. The decision-making step. Depending on the intervention priority evaluations, the manager is in a position to propose a project calendar that leads to project scheduling and execution. The description of the performed works, along with the project startup and completion dates, have been recorded in the SINERGIE's intranet information system in order to know the facility conditions at any point versus time.

The scheduling step corresponds to an optimization procedure for executing tasks on the basis of their durations and costs, which also involves related constraints of all types [58]. Road surface repair projects, for example, cannot be scheduled during the summer period given the traffic intensity experienced during this time of year. The decision-maker holds the possibility to set a strategy using "objective" functions (e.g. complete as many $P_{1}$ category projects as possible, or as many total projects as possible) and constraints (e.g. spending the budget allocation evenly throughout the year, project payment). The hierarchical multi criteria evaluation has enabled us to break down the decision-making process into several functional steps: informa-

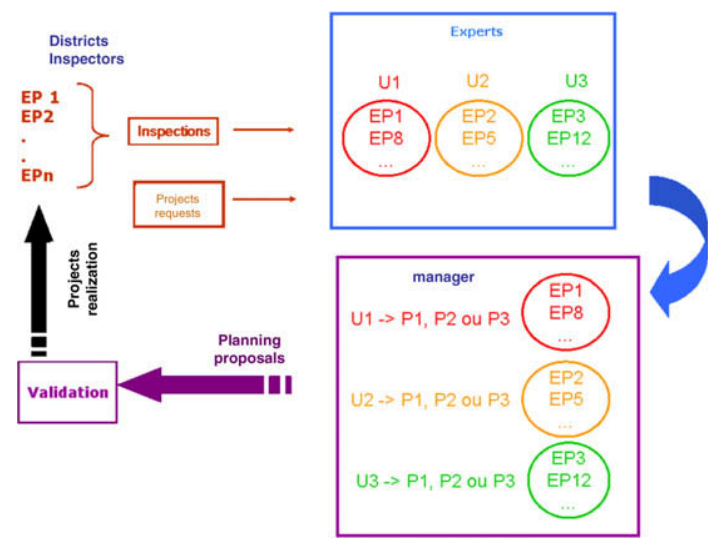

Fig. 17. Evaluation cycle of projects. 
tion processing from the measurement campaign through the project planning has been distributed. Expressing the planning function in an equation form has been facilitated by characterizing the projects in terms of intervention priority. The three steps of diagnostic, urgency evaluation and priority determination constitute the information processing phases critical to explaining the decision-making rationale of the network maintenance.

\section{Elucidation of choices and control of assessment errors}

\subsection{Justification}

A decision support system must be able to justify a decision strategy, to argue its decisional logic. Decision elucidation plays a key role in the decision acceptability [32-34,36,37].

This function offers explanation elements to the decision-making rationale at each functional level. For each decision threshold in the project evaluation process, this function seeks the dimensions that have exerted a decisive impact on the evaluations. This step entails identifying the criteria that have influenced the overall score to the greatest extent. This notion of explanation is heavily correlated with the notion discussed above of a criterion marginal contribution.

Let us now consider a project $O P_{k}$ : this project has been ascribed the highest priority according to a strategy modelled by an aggregation operator denoted $h_{w}($.$) . By definition, this yields:$ $\forall j, h_{w}\left(O P^{k}\right) \geqslant h_{w}\left(O P^{j}\right)$. In this application, $h_{w}($.$) is the weighted$ sum (see Eq. (6)). An extension of the justification principle for nonlinear operators is offered in [33,59].

It might be pertinent to begin by providing elements regarding the absolute priority of $O P_{k}$ : this constitutes a justification in absolute terms. It should then be possible to parameterize the level of required explanatory detail. Several levels of detail may in fact be sought to justify the result. A "one word" justification may be desired that cuts straight to the heart of the reasons for this preference, in offering the primary rationale, detailed justifications, or anecdotal insights.

In order to achieve this ranking in explanation terms, it is initially necessary to reorder the terms contained in the sum $\sum_{i=1}^{n} w_{i} \cdot u_{i}^{k}$, such that:

$\forall i, w_{i} \cdot u_{i}^{k} \geqslant w_{i+1} \cdot u_{i+1}^{k}$

It is then possible to segregate the absolute marginal or potential contributions in relative categories distinguished by orders of magnitude of the ratio: $\frac{w_{i} \cdot u_{i}^{k}}{w_{1} \cdot u^{k}}$. An "A $r$ B" relation is equivalent to "(A/B) $r$ 1 " and may be modelled as an interval (fuzzy, if necessary) on the ratio $(\mathrm{A} / \mathrm{B})$ by using a single parameter $e$ (the threshold $e$ features an upper limit of 0.4656 as a result of the constraint $1 / e>(1+e)^{2}$ ) [60]. As this ratio nears 1 , the contribution from criterion $i$ grows, and this criterion becomes a more essential dimension to the evaluation. A symbolic breakdown on the continuous scale provides the framework for categorizing the terms (Fig. 18).

The symbols of order-of-magnitude relations signify respectively equal to, close to, comparable with, small in comparison with and negligible in comparison with; moreover, they are associated with arguments of the type "one word", "at the heart", "mainly", "in greater detail" and "anecdotally". As an illustration, given $\frac{1}{1+e} \leqslant \frac{w_{i} \cdot u_{i}^{k}}{w_{1} u_{1}^{k}}<1$, the ratio lies near 1 , the contribution of criterion $i$ to the overall score of $O P_{k}$ provides a fundamental reason for the high overall score of $O P_{k}$.

SINERGIE is thus capable of restoring the determinant criteria in each evaluation with a level of precision that can be parameterized. The number of categories parameter has to be set and depends on the knowledge desired for dissemination (details and

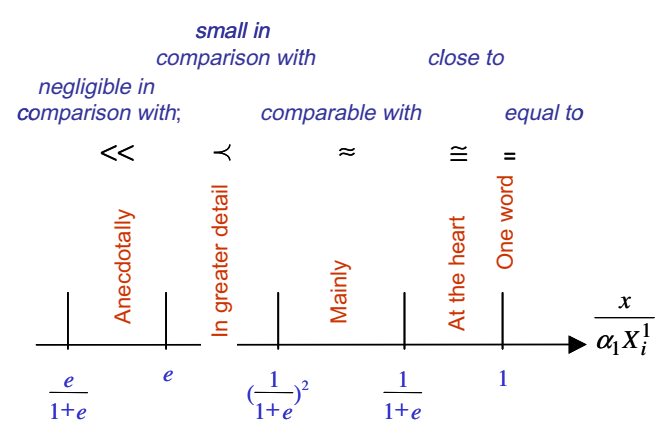

Fig. 18. Order of magnitude symbolic labels for marginal contributions.

anecdotes could for example be merged into a single linguistic category). The parameter $e$ needs to be determined, with a common value of $e$ being 0.1 , and corresponds to the shared conception that one magnitude becomes negligible in comparison with another once their ratio drops below $1 / 10$.

Another approach in considering the level of justification would be to set the percentage $\beta \%$ of $O P_{k}$ score explanation and to seek $p_{0} / h_{w}\left(O P^{k}\right) \triangleq \sum_{i=1}^{p_{0}<p} w_{i} \cdot u_{i}^{k}$,with $u_{i}^{k}$ values having been reordered as per (12).

Afterwards, during the decision legitimization step, it is important to provide answers regarding the dimensions according to which $O P_{k}$ has been given a higher priority classification than $O P_{j}$, and the dimensions on which $O P_{k}$ could be distinguished from $O P_{j}$. This generates a relative justification, where the magnitudes to be analyzed are the sums of the individual relative potentials $P_{R}^{k j}(i)=w_{i} \cdot\left(u_{i}^{k}-u_{i}^{j}\right)$ :

$\forall j, h_{w}\left(O P^{k}\right)-h_{w}\left(O P^{j}\right)=\sum_{i=1}^{p} w_{i} \cdot\left(u_{i}^{k}-u_{i}^{j}\right)=\sum_{i=1}^{p} P_{R}^{k j}(i)$

The previous rationale can now be reapplied to the orders of magnitude on this expression, after having conducted a permutation of indices $\sum_{i=1}^{p} P_{R}^{k j}(i)$ for each project $O P_{j}$ in order to sort the individual relative potentials in decreasing order.

As the final and most comprehensive level of argumentation, it might be worthwhile to position the priority project $O P_{k}$ with respect to the set of $n-1$ other projects $\left(O P_{j}\right)$ from an overall point of view.

Once again, the justification is relative, yet in this instance in comparison with projects contained in their same set, which allows relying upon the analytical criterion that has been rewritten as follows:

$\sum_{j \neq k} \sum_{i=1}^{p} w_{i} \cdot\left(u_{i}^{k}-u_{i}^{j}\right)=\sum_{i=1}^{p}\left[(n-1) u_{i}^{k}-\sum_{j \neq k} u_{i}^{j}\right] \cdot w_{i}=\sum_{i=1}^{p}\left[P_{\bar{R}}^{k}(i)\right]$

Given this expression, the problem is reduced to one similar to the previous interpretation as a function of orders of magnitude. The terms are the average relative potentials, and the most important among them will correspond to the criteria for which project $O P_{k}$ has clearly been established as having a higher priority than the average of all the projects awaiting realization.

This justification allows circulating the determinant decisionmaking criteria up to each functional level of the evaluation process: intervention priorities are thereby justified by the most critical dimensions of the entire hierarchical multi criteria evaluation. The manager is then capable of responding at any time on the maintenance policy implemented for facilities using the interactive aid system that ensures transparency of the adopted strategy.

If upon entering a partial score relative to one of these criteria, a comment (or an attachment) has been introduced to substantiate the evaluation given in a natural language, then such a comment 
will be automatically proposed as a rhetorical argument during the justification request.

Fig. 19 depicts the graphical interface of the previous justification procedure. A representation of marginal contributions from the set of criteria involved is also proposed. The project highlighted in this illustration corresponds to our "road surface A808 between kilometre posts 24 and 39" example: at a simple glance, it is possible to know the criteria that led to classifying this project in terms of both urgency $U_{2}$ and priority $P_{2}$. The result of the explanation function applied to the urgency monitoring sheet in Fig. 2 is shown in Fig. 19. The purpose is to present which criteria are responsible on $\beta \%$ (75\%) for the overall score of the urgency evaluation. The first pie chart representation highlights that safety and durability criteria are responsible for more than $63 \%$ of the overall evaluation in $U_{2}$ (first pie chart in Fig. 19). Concerning the manager risk analysis of the priority, the most discriminating criteria are safety, durability and financial (second pie chart in Fig. 19). This functionality thus helps the actors, and the company, to justify and keep the memory of their decisions.

\subsection{Control and Robustness analysis of the evaluation phase}

Let us now consider a last step in the evaluation process for preventive maintenance: assessment of the risk of erroneous estimation w.r.t. the urgency (resp. priority) of a project, i.e., the risk of underestimation or overestimation of the aggregated urgency (resp., priority) score of a project. It relies on a robustness analysis of the evaluation procedure based upon the WAM. Two aims are assigned to this step: it must answer the following questions [61]:

- when an erroneous partial estimation is done w.r.t. criterion $i$, what is the risk for the aggregated urgency degree to be affected?

- when a project appears to be underestimated (resp. overestimated), which criteria could most likely explain this faulty result?

The first question corresponds to an a priori risk estimation of erroneous evaluation; the second question is related to a diagnosis analysis.

Let us first define the notion of neighbourhood of a vector of urgency degrees $\left[U_{\left.k(1), . ., U_{k(n)}\right]}\right.$ associated to a project $O P$. The vectors of the neighbourhood of $\left[U_{\left.k(1), . ., U_{k(n)}\right]}\right.$ are all the vectors $\left[U_{k(1)}^{\prime}, \ldots, U_{k(n)}^{\prime}\right]$ such that: $\forall i \in\{1 . . n\}, U_{k(i)}^{\prime}=U_{k(i)}$ or $U_{k(i)}^{\prime}$ is the value just above (resp. below) $U_{k(i)}$ (when defined; indeed, there is no value below zero and no value above $U_{1}$ ). The neighbourhood is a set of vectors denoted $N\left(\left[U_{k(1)}, \ldots, U_{k(n)}\right]\right)$. In the example in $2 \mathrm{D}$ in Fig. 20, $U_{k(1)}=U_{2}$ and $U_{k(2)}=U_{2}$. The values of component $i(i=1$ or 2 ) of a neighbours vector may be $U_{2}, U_{1}$ or $U_{3}$. There are 8 neighbours. In the general case, the maximal number of neighbours is $3^{n}-1$.

\subsubsection{Risk of erroneous estimation}

The risk of misclassification of a project due to an overestimation (resp. underestimation) w.r.t. a criterion $i$ enables the expert in charge of a domain to assess the impact of an evaluation error w.r.t. criterion $i$ on the overall urgency degree of the project. The higher is this risk, the more carefully the partial appraisal w.r.t. criterion $i$ must be carried out. The lower is this risk, the weaker is the impact of this criterion to the global urgency degree. The risk analysis is based upon the following algorithm. We will first consider the risk of underestimation for sake of simplicity. We consider that a value $U_{k(i)}$ is underestimated (resp. overestimated) when it should take the value just above $U_{k(i)}$ (resp., just below $U_{k(i)}$ ). This assumption means that the worst appraisal error w.r.t. one criterion can only correspond to the value just below or just above for this criterion.

Let's consider a vector $U=\left[U_{k(1)}, \ldots, U_{k(n)}\right]$

Compute WAM(U)

For each criterion $i$ :

- Find all the vectors $U^{\prime}=\left[U_{k(1)}^{\prime}, \ldots, U_{k(n)}^{\prime}\right]$ in $N(U)$ such that $U_{k(i)}^{\prime}$ takes the value just above $U_{k(i)}$ (when defined, else $U_{k(i)}=U_{1}$ and there is no risk of underestimation w.r.t. criterion $i$ in this case). Note this set: Under $\_i(U)$

- Count the numbers of vectors $U^{\prime}$ in $\operatorname{Under} i(U)$ such that $\operatorname{WAM}\left(U^{\prime}\right)$ is higher than $\operatorname{WAM}(U)$. Note this number $n_{\text {under }}$

- The risk of underestimation induced by criterion $i$ for a project characterized by $U$ is then: $\operatorname{risk\_ under}(i)=$ $\frac{n_{\text {under } i} i}{\mid \text { Under } i(U) \mid}$.

In the example in Fig. 20, let us consider an assumption of underestimation w.r.t. criterion 1 . The set Under_1 $1\left(U_{2}, U_{2}\right)$ is represented in the figure. $\mid$ Under $1\left(U_{2}, U_{2}\right) \mid=3$; only $\left(U_{1}, U_{2}\right)$ and $\left(U_{1}, U_{1}\right)$ lead to an overall underestimation (the project is evaluated $U_{2}$
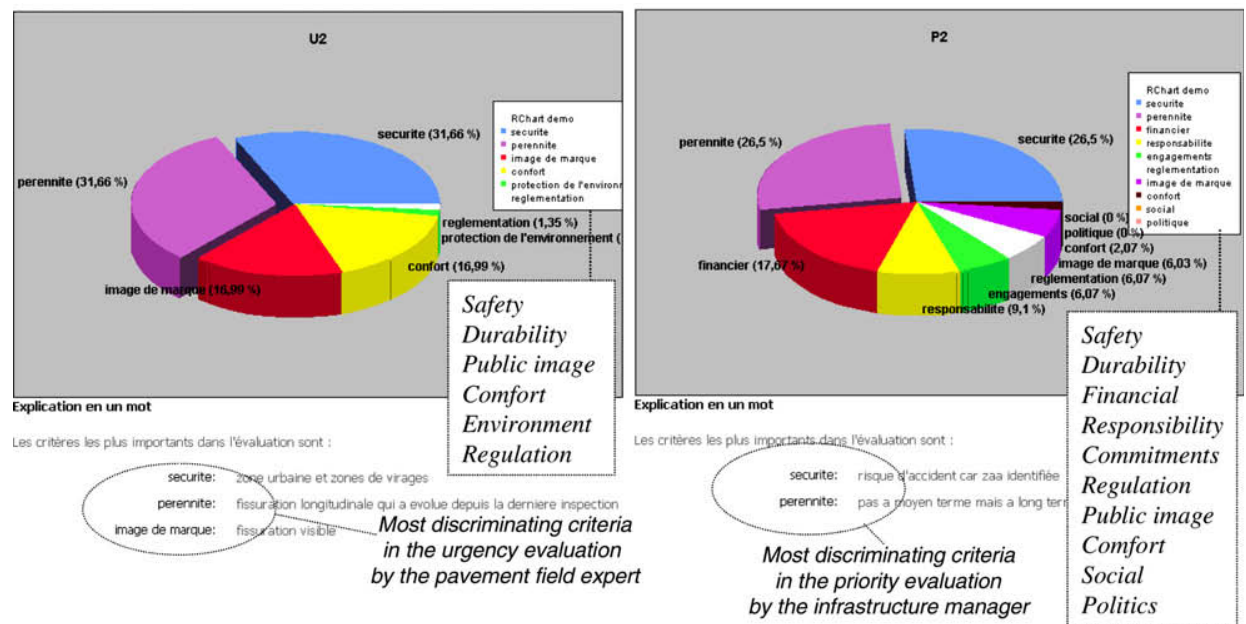

Fig. 19. "Road surface A808 between kilometre posts 24 and 39" project justification. 


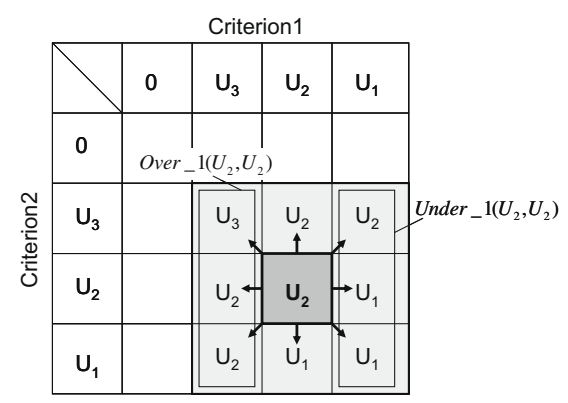

Fig. 20. Neighbourhood of the vector of urgency degrees $\left(U_{2}, U_{2}\right)$ in $2 \mathrm{D}$.

whereas it should be $\left.U_{1}\right)$. Then, $n_{\text {under_1 }}=2$ and risk_under $(1)=$ $2 / 3$. It means that an underestimation w.r.t. criterion 1 for a project characterized by $\left(U_{2}, U_{2}\right)$ leads to an underestimation of the overall degree of urgency of the project in $66 \%$ of the cases.

The algorithm is the same for the risk of overestimation. Nevertheless, in this case, when $U_{k(i)}=0$, the risk of overestimation w.r.t. criterion $i$ is null.

Figs. 21 and 22 provide the results for the risk of erroneous estimation when underestimation (Fig. 21) and when overestimation (Fig. 22) for all the road surface projects in Fig. 15. For example, for project 21 (Fig. 21), an underestimation error w.r.t. criterion environment will lead to an underestimation of the global urgency degree attributed to project 21 in $44 \%$ of the cases. For project 21 (Fig. 22), an overestimation error w.r.t. criterion environment will lead to an overestimation of the global urgency degree attributed to project 21 in only $3 \%$ of the cases.

The man machine interface in Fig. 25-left part shows the graphical representation generated with SINERGIE w.r.t. to the "road surface A808 between kilometre posts 24 and 39" urgency evaluations (Fig. 9). In the top left graphic (Fig. 25), underestimation on security criterion is responsible for overall underestimation of the urgency evaluation in $75 \%$ of the cases. This result is rather intuitive because the relative importance of security criterion is significant in this example. This kind of result is a particularly useful feature when the expert hesitates on an assessment w.r.t. a criterion: indeed, this risk analysis highlights the possible consequences that might result from an error w.r.t. a criterion. This does not mean that the expert is wrong in his evaluation but he knows the consequences upon his decision due to an erroneous estimation w.r.t. this criterion. As a consequence, he can better monitor his analysis: if he considers that the risk is too high he can engage further investigations w.r.t. the risky criterion before he gives his final assessment. On the contrary, if the risk of overall underestimation (resp. overestimation) induced by a partial erroneous assessment is weak, it is not necessary to pay a particular attention to the criterion and no further investigation will be engaged w.r.t. this criterion: the risk of erroneous overall evaluation is then negligible.

\subsubsection{Diagnosis analysis}

When the degree of urgency of a project is suspected to be overestimated (resp. underestimated), the diagnosis analysis consists in determining the most likely causes, i.e., the criteria that the most frequently lead to an overestimation (resp. underestimation) of the project when they are, themselves, overestimated (resp. underestimated). The possibility that criterion $i$ is a cause of overestimation (resp. underestimation) assuming an overestimation (resp. underestimation) of the overall urgency degree of the project is computed in the diagnosis step.

Let us consider the algorithm in case of underestimation (resp., overestimation).

Let's consider a vector $U=\left[U_{k(1)}, \ldots, U_{k(n)}\right]$

Compute WAM(U)

Compute $\mathrm{N}(\mathrm{U})$ and its cardinal $|\mathrm{N}(\mathrm{U})|$

- Compute $\operatorname{WAM}\left(U^{\prime}\right)$ for each $U^{\prime}=\left[U_{k(1)}^{\prime}, \ldots, U_{k(n)}^{\prime}\right]$ in $\mathrm{N}(\mathrm{U})$

- Let us note Higher_N(U) (resp. Lower $N(U)$ ), the set of vectors $U^{\prime}$ in $N(U)$ such that $\operatorname{WAM}\left(U^{\prime}\right)>\operatorname{WAM}(U)$ (resp. $\left.\operatorname{WAM}\left(U^{\prime}\right)<\operatorname{WAM}(U)\right)$

- For each criterion $i$, count the number $n_{u n d e r \_}^{\prime}\left(\right.$ resp. $\left.n_{\text {over_i }}^{\prime}\right)$ of times criterion $i$ is underestimated (resp. overestimated) in a vector of Higher $N(U)$ (resp. Lower $N(U)$ ), i.e., $U_{k(i)}^{\prime}$ takes the value just above $U_{k(i)}$ (resp. just below $\left.U_{k(i)}\right)$ in Higher $\_(U)$ (resp. Lower $\_(U)$ )

- Compute for each criterion $i$ : Diag_under $(i)=\frac{n_{n^{\prime}}^{\prime}{ }_{\text {under } i}}{\mid \text { Higher_N(U) }_{\text {_ }} \mid}$ (resp. Diag_over $(i)=\frac{n_{\text {over } i} i}{\left.\left|\operatorname{Lower} \_\mathrm{N}(U)\right|_{\text {ove }}\right)}$.

Diag_under $(i)$ gives the rate that an underestimation w.r.t. criterion $i$ is a potential cause of underestimation of the overall urgency degree of a project (idem for overestimation).

Fig. 23 concerns underestimation diagnosis and Fig. 24 overestimation diagnosis for the data base of the road surface projects in Fig. 15. A rate indicates the probability for a criterion to be underestimated itself (resp., overestimated) when the overall urgency degree of the concerned project is underestimated (resp. overestimated). For example, for project 21, an underestimation w.r.t. criterion security is a cause of underestimation of the overall urgency degree of the project in $53 \%$ of the cases.

This analysis applied to the "road surface A808 between kilometre posts 24 and 39" urgency evaluation (Fig. 9) generates the results shown in Fig. 25-right part. The top-right graphic can be read as follows: may the overall urgency score be underestimated (i.e., the overall score should be $U_{1}$ instead of $U_{2}$ ), then underestimation on security criterion shall be responsible in $65 \%$ of underestimation cases (Fig. 25 top-right). If the manager has some doubts concerning the diagnosis of his expert, he can suggest checking security criterion assessment as a priority. Besides, still considering the $U_{2}$ urgency evaluation in Fig. 9, a $U_{3}$ overall estimation would be possible if and only if all criteria were valued $U_{3}$. Hence, the results on the bottom-right graphic show that influence criteria are merely the four $U_{2}$ valued criteria in Fig. 9. To be overall overestimated, it means that the expert should be wrong on at least 4 criteria; the manager can reasonably consider this situation as unlikely w.r.t. the evaluation form. In this case, the manager can

\begin{tabular}{|c|c|c|c|c|c|c|c|c|c|c|c|c|c|c|c|c|c|c|c|c|c|c|}
\hline & 17 & $21 \mid$ & 19 & 30 & 12 & 11 & 29 & 15 & 14 & 27 & 28 & 13 & 6 & 9 & 8 & 7 & 25 & 24 & 23 & 22 & 5 & 18 \\
\hline env & $11.0 \%$ & $44.0 \%$ & $0 \%$ & $0 \%$ & $15.0 \%$ & $2.0 \%$ & $0 \%$ & $0 \%$ & $0 \%$ & $26.0 \%$ & $13.0 \%$ & $41.0 \%$ & $0 \%$ & $0 \%$ & $0 \%$ & $0 \%$ & $18.0 \%$ & $21.0 \%$ & $0 \%$ & $0 \%$ & $0 \%$ & \begin{tabular}{|l|l|}
$0 \%$ & $21.0 \%$ \\
\end{tabular} \\
\hline sanitary & $11.0 \%$ & $42.0 \%$ & $0 \%$ & $0 \%$ & $14.0 \%$ & $1.0 \%$ & $0 \%$ & $0 \%$ & $0 \%$ & $26.0 \%$ & $13.0 \%$ & $39.0 \%$ & $0 \%$ & $0 \%$ & $0 \%$ & $0 \%$ & $18.0 \%$ & $21.0 \%$ & $0 \%$ & $0 \%$ & $0 \%$ & $0 \% 21.0 \%$ \\
\hline comfort & $25.0 \%$ & $58.0 \%$ & $0 \%$ & $0 \%$ & $25.0 \%$ & $4.0 \%$ & $0 \%$ & $0 \%$ & $0 \%$ & $40.0 \%$ & $27.0 \%$ & $55.0 \%$ & $0 \%$ & $0 \%$ & $0 \%$ & $0 \%$ & $28.0 \%$ & $32.0 \%$ & $0 \%$ & $0 \%$ & $0 \%$ & \begin{tabular}{|l|l|}
$0 \%$ & $37.0 \%$ \\
\end{tabular} \\
\hline regulation & $11.0 \%$ & $45.0 \%$ & $0 \%$ & $0 \%$ & $16.0 \%$ & $2.0 \%$ & $0 \%$ & $0 \%$ & $0 \%$ & $34.0 \%$ & $20.0 \%$ & $43.0 \%$ & $0 \%$ & $0 \%$ & $0 \%$ & $0 \%$ & $20.0 \%$ & $25.0 \%$ & $0 \%$ & $0 \%$ & $0 \%$ & \begin{tabular}{l|l}
$0 \%$ & $23.0 \%$ \\
\end{tabular} \\
\hline security & $32.0 \%$ & $67.0 \%$ & $0 \%$ & $0 \%$ & $37.0 \%$ & $4.0 \%$ & $0 \%$ & $0 \%$ & $0 \%$ & $62.0 \%$ & $35.0 \%$ & $76.0 \%$ & $0 \%$ & $0 \%$ & $0 \%$ & $0 \%$ & $41.0 \%$ & $46.0 \%$ & $0 \%$ & $0 \%$ & $0 \%$ & \begin{tabular}{|l|l|}
$0 \%$ & $55.0 \%$ \\
\end{tabular} \\
\hline durability & $15.0 \%$ & $73.0 \%$ & $0 \%$ & $0 \%$ & $28.0 \%$ & $4.0 \%$ & $0 \%$ & $0 \%$ & $0 \%$ & $32.0 \%$ & $17.0 \%$ & $62.0 \%$ & $0 \%$ & $0 \%$ & $0 \%$ & $0 \%$ & $39.0 \%$ & $44.0 \%$ & $0 \%$ & $0 \%$ & $0 \%$ & \begin{tabular}{|l|l|}
$0 \%$ & $27.0 \%$ \\
\end{tabular} \\
\hline social & $11.0 \%$ & $42.0 \%$ & $0 \%$ & $0 \%$ & $14.0 \%$ & $1.0 \%$ & $0 \%$ & $0 \%$ & $0 \%$ & $26.0 \%$ & $13.0 \%$ & $39.0 \%$ & $0 \%$ & $0 \%$ & $0 \%$ & $0 \%$ & $18.0 \%$ & $21.0 \%$ & $0 \%$ & $0 \%$ & $0 \%$ & \begin{tabular}{l|l|l}
$0 \%$ & $21.0 \%$ \\
\end{tabular} \\
\hline public image & $18.0 \%$ & $56.0 \%$ & $0 \%$ & $0 \%$ & $26.0 \%$ & $4.0 \%$ & $0 \%$ & $0 \%$ & $0 \%$ & $44.0 \%$ & $27.0 \%$ & $58.0 \%$ & $0 \%$ & $0 \%$ & $0 \%$ & $0 \%$ & $29.0 \%$ & $34.0 \%$ & $0 \%$ & $0 \%$ & $0 \%$ & $\begin{array}{l}0 \% 39.0 \% \\
\end{array}$ \\
\hline
\end{tabular}

Fig. 21. Risk of overall underestimation (resp., overestimation) of the projects induced by partial underestimations (resp., overestimations) w.r.t. criteria. 


\begin{tabular}{|c|c|c|c|c|c|c|c|c|c|c|c|c|c|c|c|c|c|c|c|c|c|c|c|}
\hline & 17 & 21 & 19 & 30 & 12 & 11 & 29 & 15 & 14 & 27 & 28 & 13 & 6 & 9 & 8 & 7 & 25 & 24 & 23 & 22 & 5 & 2 & 18 \\
\hline env & $2.0 \%$ & $3.0 \%$ & $0.0 \%$ & $53.0 \%$ & $7.0 \%$ & $30.0 \%$ & $12.0 \%$ & $.0 \%$ & $17.0 \%$ & $1.0 \%$ & $6.0 \%$ & $0.0 \%$ & $41.0 \%$ & $34.0 \%$ & $34.0 \%$ & $28.0 \%$ & $13.0 \%$ & $1.0 \%$ & $.0 \%$ & $6.0 \%$ & $0.0 \%$ & $0.0 \%$ & $1.0 \%$ \\
\hline sanitary & $2.0 \%$ & $3.0 \%$ & $0.0 \%$ & $53.0 \%$ & $6.0 \%$ & $29.0 \%$ & $12.0 \%$ & $16.0 \%$ & $16.0 \%$ & $1.0 \%$ & $6.0 \%$ & $0.0 \%$ & $34.0 \%$ & $4.0 \%$ & $34.0 \%$ & $.0 \%$ & $13.0 \%$ & $.0 \%$ & $.0 \%$ & $6.0 \%$ & $0 \%$ & $0.0 \%$ & $1.0 \%$ \\
\hline comfort & $8.0 \%$ & $7.0 \%$ & $1.0 \%$ & $69.0 \%$ & $13.0 \%$ & $46.0 \%$ & $19.0 \%$ & $24.0 \%$ & $24.0 \%$ & $3.0 \%$ & $14.0 \%$ & $2.0 \%$ & $51.0 \%$ & $51.0 \%$ & $51.0 \%$ & $39.0 \%$ & $20.0 \%$ & $18.0 \%$ & $12.0 \%$ & $12.0 \%$ & $2.0 \%$ & $1.0 \%$ & $3.0 \%$ \\
\hline regulation & $3.0 \%$ & $3.0 \%$ & $1.0 \%$ & $58.0 \%$ & $9.0 \%$ & $32.0 \%$ & $19.0 \%$ & $23.0 \%$ & $23.0 \%$ & $1.0 \%$ & $6.0 \%$ & $1.0 \%$ & $40.0 \%$ & $35.0 \%$ & $35.0 \%$ & $28.0 \%$ & $14.0 \%$ & $14.0 \%$ & $7.0 \%$ & $7.0 \%$ & $1.0 \%$ & $0.0 \%$ & $1.0 \%$ \\
\hline security & $8.0 \%$ & $9.0 \%$ & $1.0 \%$ & $72.0 \%$ & $18.0 \%$ & $63.0 \%$ & $35.0 \%$ & $47.0 \%$ & $47.0 \%$ & $3.0 \%$ & $18.0 \%$ & $2.0 \%$ & $74.0 \%$ & $80.0 \%$ & $80.0 \%$ & $56.0 \%$ & $30.0 \%$ & $27.0 \%$ & $19.0 \%$ & $19.0 \%$ & $2.0 \%$ & $1.0 \%$ & $3.0 \%$ \\
\hline \begin{tabular}{|l|} 
durability \\
\end{tabular} & $8.0 \%$ & $9.0 \%$ & $1.0 \%$ & $96.0 \%$ & $17.0 \%$ & $49.0 \%$ & $24.0 \%$ & $30.0 \%$ & $30.0 \%$ & $3.0 \%$ & $11.0 \%$ & $2.0 \%$ & $48.0 \%$ & $48.0 \%$ & $48.0 \%$ & $36.0 \%$ & $39.0 \%$ & $34.0 \%$ & $18.0 \%$ & $18.0 \%$ & $2.0 \%$ & $1.0 \%$ & $3.0 \%$ \\
\hline social & $2.0 \%$ & $3.0 \%$ & $0.0 \%$ & $53.0 \%$ & $6.0 \%$ & $29.0 \%$ & $12.0 \%$ & $16.0 \%$ & $16.0 \%$ & $1.0 \%$ & $6.0 \%$ & $0.0 \%$ & $34.0 \%$ & $34.0 \%$ & $34.0 \%$ & $22.0 \%$ & $13.0 \%$ & $11.0 \%$ & $6.0 \%$ & $6.0 \%$ & $0.0 \%$ & $0.0 \%$ & $1.0 \%$ \\
\hline public image & $3.0 \%$ & $5.0 \%$ & $1.0 \%$ & $60.0 \%$ & $15.0 \%$ & $44.0 \%$ & $24.0 \%$ & $29.0 \%$ & $29.0 \%$ & $3.0 \%$ & $14.0 \%$ & $2.0 \%$ & $53.0 \%$ & $48.0 \%$ & $48.0 \%$ & $33.0 \%$ & $20.0 \%$ & $18.0 \%$ & $12.0 \%$ & $12.0 \%$ & $2.0 \%$ & $1.0 \%$ & $3.0 \%$ \\
\hline
\end{tabular}

Fig. 22. Risk of overall underestimation (resp. overestimation) of the projects induced by partial underestimations (resp. overestimations) w.r.t. criteria.

practically eliminate any overestimation error. This kind of results is of use for the manager to suggest further precise and quantitative investigations (or not) w.r.t. suspicious criteria.

\subsection{Control interpretation}

Let us now consider the way the influence of an erroneous partial criterion score may affect the final ranking of projects for planning purposes. Indeed, there is a risk that a partial overestimation or underestimation is sufficient to modify the final planning of the projects. This decisional risk can be seen as related to the reliability of the evaluation process of projects by experts and managers. Our risk notion can be seen from a conventional probabilistic point of view (erroneous score probability multiplied by the associated gravity, i.e., the impact upon the planning year) but is more consistent with a sensitivity analysis of the projects ranking that is itself linked to the sensitivity of the aggregated scores to partial score variations. We preferred to associate the decisional risk concept to a sensitivity analysis rather than to a robustness analysis. We can say that robustness is generally connected to the fact that decision-aid methods often contain parameters whose values have to be chosen (more or less arbitrarily) by the user. Intuitively, a solution will be considered robust if the results obtained for different plausible values of the parameters do not contradict each other. Following this line, several studies are available in the literature [62-64]. In our case, the sensitivity analysis is not concerned with the influence of the parameters on the decision result, but is focused on the sensitivity of the aggregated score to any variations of the partial scores (see examples in previous Sections 4.2.1 and 4.2.2).

The evaluation errors assessments can then be used to control the decision process as intuitively introduced in the above examples. Indeed, as soon as, one of the field experts doubts the accuracy of one of his partial evaluations (i.e., he considers the risk of misclassification of a project, due to an overestimation or an underestimation, being too high (Section 4.1.)) or the manager suspects the facility expert diagnosis of inaccuracy (i.e., he strongly suspects the overall degree of urgency of a project to be overestimated or underestimated (Section 4.2)), further inspections or advanced technical measurements, more quantitative and precise investigations are required to get a more reliable re-evaluation. The risk of erroneous evaluation thus appears as a control variable of the decision-making process relative to projects planning. It enables to detect the more suspicious criteria, the riskiest ones, in the evaluation process.

This risk notion is related to the acceptability of decisions. An error of judgment - i.e. selecting a project that would later turn out to be a regrettable choice due to an unjustified alarm - is related to the accuracy of knowledge available to decide at that time (accuracy of diagnoses, inspections results, etc.). It corresponds to an epistemic uncertainty, i.e., an uncertainty related to the state of the available precise knowledge at the current time. At that time, the state of knowledge determines the reliability of the decision. When a small additional amount of new quantitative and accurate data is sufficient to modify the classification of a project, the epistemic uncertainty is high. The risk of erroneous evaluation that is related to the sensitivity of the project classification simply captures this concept of epistemic incertitude: the more suspicious the expert or the infrastructure manager, the riskier the project classification.

Another way to introduce the risk of error concept as a control variable is the following. It is merely introduced to capture the knowledge base entropy ${ }^{8}$ at stake in the decision process: when the risk is weak, the quantity of information (further quantitative investigations, more precise diagnoses) necessary to modify the project classification should be significant, and the classification is reliable. When the risk is high, a minor quantity of accurate and precise information could modify the project classification: it is then unstable. The risk is thus related to the reliability of the decision, and the reliability is a function of the information accuracy, thus the decisional risk concept can be considered as an observer of the internal entropy of the data base of SINERGIE information system.

Finally, these viewpoints find a place in a cybernetic interpretation (Fig. 26) of Simon IDCR representation we mentioned in Section 2 of this paper (Fig. 2). The risk of misclassification is the control variable, the controlled process is the hierarchical multi criteria evaluation process and the set point of this control loop is the decision acceptability level (threshold).

This reasoning framework for the projects planning decisional process based on a prescriptive view of Simon's model is an analogy with a control theory representation. The cognitive loop of Simon's model - the review phase - that completed his InformationDesign-Choice model is interpreted in terms of feedback loops.

\section{Conclusion}

Infrastructure aging is a constant concern for facility managers, who must be in a position to guarantee user safety and effective facility operations over time. Confronted with a massive amount of information to process, it becomes vital to define a durable decision aid strategy that assists managers in both their decision-making and legitimization of investment rationale.

ESCOTA has invested in a multifaceted approach involving: measurement, evaluation and decision-making. The motorway company is now equipped with a computerized decision aid tool for the purpose of improving both maintenance and facility management: the SINERGIE software. This application is composed of an information system (IS) and interactive decision aid system to help operational actors in project scheduling of maintenance, retrofitting and upgrading of the various network elements. The decision process encompasses a multi-actor and multi criteria aspect (inspectors, experts, the manager). The evaluations depend on each field and level of individual responsibility, along with the analytical criteria associated with these fields and levels. The decision aid system proposed by the software can be used as a tool to legitimize the management policy. Breaking down project evaluation in terms of diagnostic, urgency and

\footnotetext{
${ }^{8}$ The notion of entropy is chosen here by analogy with the Theory of Information. But here, the entropy is a function of the rate of the potential decisions before and after the message delivery. It corresponds to the degree of indetermination in the communication.
} 


\begin{tabular}{|c|c|c|c|c|c|c|c|c|c|c|c|c|c|c|c|c|c|c|c|c|c|c|c|}
\hline & 17 & 21 & 19 & 30 & 12 & 11 & 29 & 15 & 14 & 27 & 28 & 13 & 6 & 9 & 8 & 7 & 25 & 24 & 23 & 22 & 5 & 2 & 18 \\
\hline urability & $5 \%$ & $57 \%$ & $0 \%$ & $0 \%$ & $67 \%$ & $0 \%$ & $0 \%$ & $0 \%$ & $0 \%$ & $40 \%$ & $42 \%$ & $52 \%$ & $0 \%$ & $0 \%$ & $0 \%$ & $0 \%$ & $71 \%$ & $67 \%$ & $0 \%$ & $0 \%$ & $0 \%$ & $0 \%$ & $41 \%$ \\
\hline ecu & $\%$ & & $8 \%$ & 8 & $\%$ & & & & & & $87 \%$ & $4 \%$ & & & & $0 \%$ & $75 \%$ & $1 \%$ & & $\Omega$ & $\%$ & $\%$ & $4 \%$ \\
\hline or & $\%$ & $\%$ & & & $9 \%$ & $90 \%$ & & & $\%$ & $\%$ & $66 \%$ & $46 \%$ & & & $0^{\circ}$ & $0 \%$ & $1 \%$ & $49 \%$ & & & $0 \%$ & $\%$ & $6 \%$ \\
\hline public & $\%$ & $4 \%$ & $0^{\circ}$ & & $2 \%$ & & & & 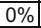 & $\% \%$ & $66^{\circ}$ & $18 \%$ & & & $0^{\circ}$ & $0 \%$ & $3 \%$ & $52^{\circ}$ & & & $0^{\circ}$ & $\%$ & $0 \%$ \\
\hline env & $35 \%$ & $35 \%$ & $0 \%$ & $0 \%$ & $36 \%$ & $45 \%$ & $0 \%$ & $0 \%$ & $0 \%$ & $3 \%$ & $33 \%$ & $35^{\circ}$ & $0 \%$ & 0 & $0 \%$ & $0 \%$ & $33 \%$ & 33 & 0 & 0 & 07 & $0 \%$ & $3 \%$ \\
\hline egula & $35 \%$ & $35 \%$ & $0 \%$ & $0 \%$ & $37 \%$ & $45 \%$ & $0 \%$ & $0 \%$ & $0 \%$ & $43 \%$ & $51 \%$ & $36 \%$ & $0 \%$ & $0 \%$ & $0 \%$ & $0 \%$ & $37 \%$ & $39^{\circ}$ & $0 \%$ & $0 \%$ & $0 \%$ & $0 \%$ & $35 \%$ \\
\hline social & $33 \%$ & $33 \%$ & $0 \%$ & $0 \%$ & $33 \%$ & $33 \%$ & $0 \%$ & $0 \%$ & $0 \%$ & $33 \%$ & $33 \%$ & $33 \%$ & $0 \%$ & $0 \%$ & $0 \%$ & $0 \%$ & $33 \%$ & 33 & $0^{\circ}$ & $0^{\circ}$ & $0^{\circ}$ & $0 \%$ & $33 \%$ \\
\hline anitary & $33 \%$ & $33 \%$ & $0 \%$ & $0 \%$ & $33 \%$ & $33 \%$ & $0 \%$ & $0 \%$ & $0 \%$ & $33 \%$ & $33 \%$ & $33 \%$ & $0 \%$ & $0 \%$ & $0 \%$ & $0 \%$ & $33 \%$ & $33 \%$ & $0 \%$ & $0 \%$ & $0 \%$ & $0 \%$ & $33 \%$ \\
\hline
\end{tabular}

Fig. 23. Rates of causes of underestimation (resp., overestimation) diagnoses.

\begin{tabular}{|c|c|c|c|c|c|c|c|c|c|c|c|c|c|c|c|c|c|c|c|c|c|c|c|}
\hline & 17 & 21 & 19 & 30 & 12 & 11 & 29 & 15 & 14 & 27 & 28 & 13 & 6 & 9 & 8 & 1 & 25 & 24 & 23 & 22 & 4 & 2 & 18 \\
\hline ural & $0 \%$ & $0 \%$ & $0 \%$ & $0 \%$ & $2 \%$ & $57 \%$ & $66 \%$ & $1 \%$ & $1 \%$ & $0 \%$ & $60 \%$ & $0 \%$ & $5 \%$ & $46 \%$ & $5 \%$ & $3 \%$ & $4 \%$ & $96 \%$ & $93 \%$ & $93 \%$ & $0 \%$ & $00 \%$ & $\%$ \\
\hline & & & & & & & & & & & & & & & & & & & $100 \%$ & $0 \%$ & & & \\
\hline & & & & & & & & & & & & & & & & & & & & & & & \\
\hline & & & & & & & & & & & & & & & & & & & & & & & $\pi$ \\
\hline nv & $\%$ & $\%$ & & $33 \%$ & $\%$ & $34 \%$ & $33 \%$ & $5 \%$ & $\%$ & & & & 40 & & & & & & & & & $\%$ & $3 \%$ \\
\hline egul & $40 \%$ & $9 \%$ & $0 \%$ & $35 \%$ & $3 \%$ & $\%$ & $3 \%$ & 710 & $5 \%$ & 33 & 33 & 00 & $39 \%$ & 34 & & 12 & & & & & & & $3 \%$ \\
\hline ocia & $33 \%$ & $33 \%$ & $33 \%$ & $33 \%$ & $33 \%$ & $33 \%$ & $33 \%$ & $33 \%$ & $33 \%$ & $33^{\circ}$ & 33 & 33 & 33 & 33 & 33 & $\overline{33}$ & 33 & 33 & 3 & 100 & 33 & 33 & $3 \%$ \\
\hline anita & $3 \%$ & $33 \%$ & $33 \%$ & $33 \%$ & $33 \%$ & $33 \%$ & $33 \%$ & $3 \%$ & $33 \%$ & $33 \%$ & $33 \%$ & $3 \%$ & $33 \%$ & $33 \%$ & $33 \%$ & $3 \%$ & $3 \%$ & $33 \%$ & $33 \%$ & $3 \%$ & $33 \%$ & $3 \%$ & $33 \%$ \\
\hline
\end{tabular}

Fig. 24. Rates of causes of underestimation (resp., overestimation) diagnoses.
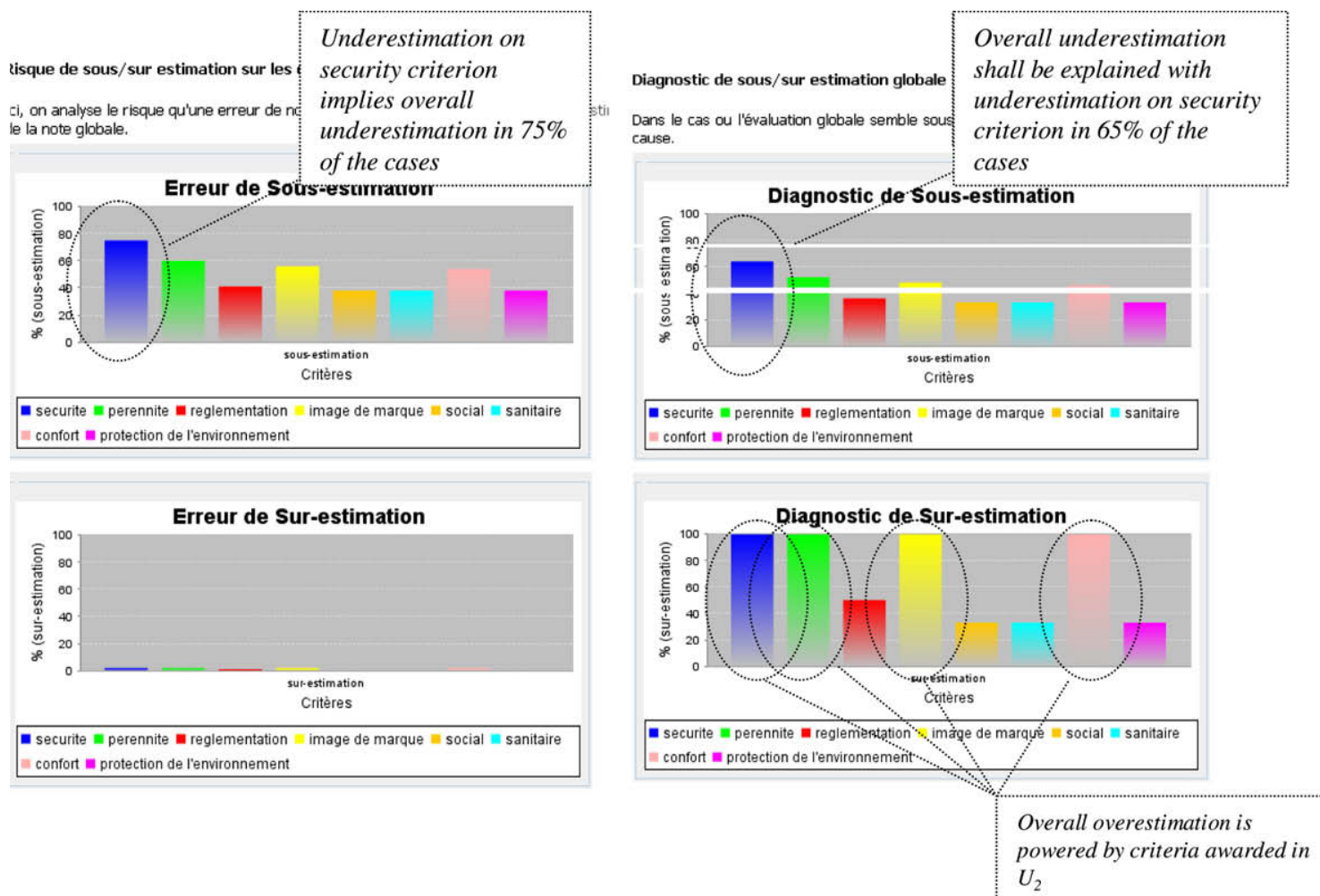

Fig. 25. "Road surface A808 between kilometre posts 24 and 39" robustness analysis.

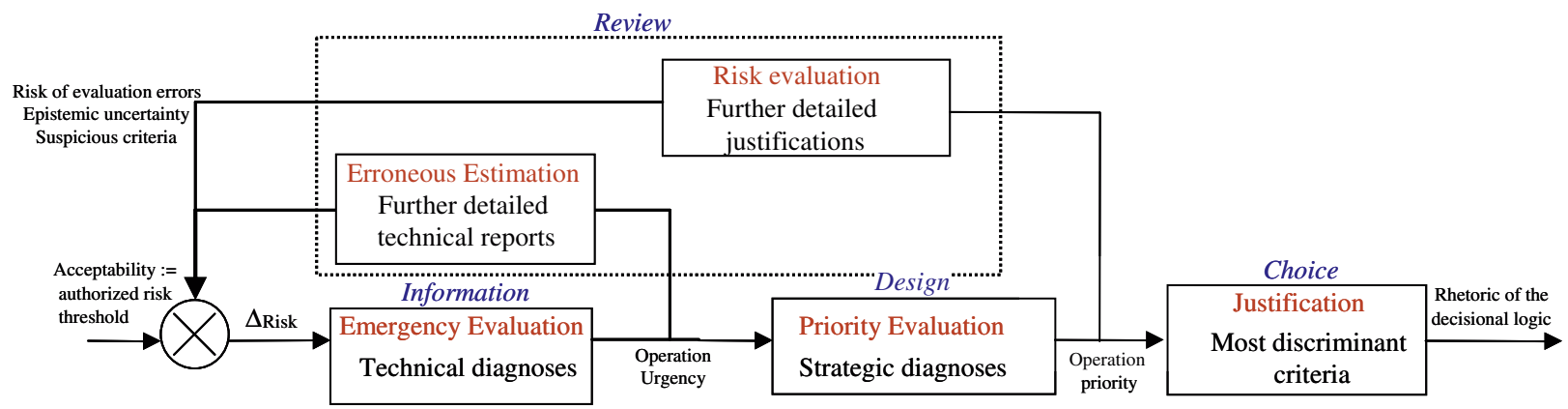

Fig. 26. Cybernetic interpretation of cognitive loops in Simon's IDCR model. 
priority constitutes a vital information processing phase that serves to facilitate and ultimately prepare a well-substantiated project plan.

All our technical choices have been guided by the application. From this point of view, a particular attention has been paid to the formalization of the multi criteria assessment process to attribute urgency and priority degrees to a maintenance operation. Aggregation procedures with discrete scales raise problems that necessitate some precautions. The problems that discrete scales cause also explain our mathematical choices regarding the sensitivity analysis of our models for robustness evaluation and explanation purposes. Nevertheless, the most esthetical way to proceed would have been to use a symbolic WAM as it is proposed in the theoretical approach in [57]: when evaluations are expressed in terms of symbolic labels over finite scales, a symbolic generalised WAM should be required. We have implemented this theoretical approach in SINERGIE. As already mentioned, these results cannot be presented here for paper length reasons, but also for practical reasons: the symbolic approach required a huge learning data base of projects and implied non common mathematical concepts for ESCOTA engineers. On the contrary, the 3-step evaluation process of a project (Fig. 16) we propose here has been accepted with no trouble by users from a practical point of view. As a conclusion, we would like to simply add that no difference was observed between both approaches when compared over learning data bases. The poor semantic universe - only 3 labels - implied in our application can partly explain such a perfect matching. Another limit of the SINERGIE project consists in the aggregation operator. The SINERGIE projects aims at formalising the evaluation process of maintenance and rehabilitation projects: too complex mathematical tools might have been an obstacle to set the SINERGIE mechanisms going and to implement the DSS in practice. This project was an initial phase: we are conscious that many refinements could be proposed. In particular, our proposals could be extended to more sophisticated aggregation models. In other works, we have proposed alike functionalities with Choquet fuzzy integrals that generalize the WAM aggregation when modelling the potential criteria interactions is a requirement $[33,34,39]$. Finally, we would like to mention that the planning of the projects is not explicitly described here: the suggested basic idea is that the higher the priority of a project is, the sooner it is planned. More complex rules are provided in [58] but cannot be provided here for paper length reasons. Nevertheless, we believe that further investigations should complete the projects planning proposals in SINERGIE: the hierarchical multi criteria assessment process we describe in this paper was the necessary formalization step that now opens new opportunities for optimisation tools dedicated to projects planning purposes in the future [65].

\section{Acknowledgements}

This Sinergie tool has been developed within the scope of the thesis work performed by Céline Sanchez through the "CIFRE" contract (an industry agreement promoting training through research, with the financial support of the French Research Ministry), which encompasses the LGI2P Laboratory (computer and production engineering), the CMGD Materials Research Center of Alès School of Mines and the ESCOTA motorway company. Special thanks to Brigitte Mahieu and André Nicolas (ESCOTA). The authors would like to thank the French National Research Agency technical branch for its financial support and extend their gratitude to the members of staff of ESCOTA Structural Viability and Safety Unit and of the company various district offices.

\section{References}

[1] J.C. Kunz, T. Tomiyama, I.F.C. Smith, (Ed.), Advanced Engineering in Informatics, vol. 22(1), Elsevier, 2008

[2] K.M. Saridakis, A.J. Dentsoras, Soft computing in engineering design - a review Advanced Engineering in Informatics 22 (2) (2008) 202-221.

[3] R. Peachavanish, H.A. Karimi, B. Akinci, F. Boukamp, An ontological engineering approach for integrating CAD and GIS in support of infrastructure management, Advanced Engineering in Informatics 20 (1) (2006) 71-88.

[4] S. Dehlin, T. Olofsson, An evaluation model for ICT investments in construction projects, ITcon, 13, Special Issue Case studies of BIM use, 2008, pp. 343-361.

[5] L.-C. Wang, Y.-C. Lin, P.H. Lin, Dynamic mobile RFID-based supply chain control and management system in construction, Advanced Engineering in Informatics 21 (4) (2007) 377-390.

[6] D. Kostoulas, R. Aldunate, F. Pena Mora, S. Lakhera, A nature-inspired decentralized trust model to reduce information unreliability in complex disaster relief operations, Advanced Engineering in Informatics 22 (1) (2008) 45-58.

[7] J. Vanier Dana, Decision support systems in infrastructure management editorial, ITcon, 11, Special Issue Decision Support Systems for Infrastructure management, 2006, pp. 175-176.

[8] Laing, R., Miller, D., Davis, A.-M, Scott,S. Urban green space: the incorporation of environmental values in a decision support system, ITcon, 11, Special Issue Decision Support Systems for Infrastructure management, 2006, pp. 177-196.

[9] Shevers, H. Demand support by virtual experts; Supporting the client during the inception phase of a building and construction project. PhD thesis, Schijndel, the Netherlands, 2004.

[10] H. Shevers, G. Trinidad, R. Drogemuller, Towards integrated assessments for urban development, ITcon, 11, Special Issue Decision Support Systems for Infrastructure management, 2006, pp. 225-236.

[11] A. Osman, R. Ries, Life cycle impact analysis of energy systems in buildings, Journal of infrastructure Systems 10 (3) (2004) 115-124. Part A.

[12] A. Osman, R. Ries, Optimization for cogeneration systems in building based on life cycle assessment, ITcon, 11, Special Issue Decision Support Systems for Infrastructure management, 2006, pp. 269-284.

[13] R.N. Palmer, A. Mohammadi, M.A. Hahn, D. Kessler, J.V. Dvorak, D. Parkinson, Computer assisted decision support system for high level infrastructure master planning: case of city of Portland supply and transmission model (STM), in: Proceedings of the ASCE 2000 Joint Conference on Water Resources Engineering and Water Resources Planning and Movement, Minneapolis, USA 2000.

[14] O.O. Ugwu, J.H.M. Tah, A turnkey client-server decision support architecture for project management, Journal of Decision Systems 8 (1999) 116.

[15] M. Moglia, S. Burn, S. Meddings, Decision support system for water pipeline renewal priorisation, ITcon, 11, Special Issue Decision Support Systems for Infrastructure management, 2006, pp. 269-284.

[16] S. Saegrov, A. König, A. Mattick, J. Milina, J. Röstum, G. Syrne, Methods for estimating water network rehabilitation needs, Water Supply 3 (2002) 6369.

[17] J. Malandain, P. Le Gauffre, M. Miramond, Organizing a decision support system for infrastructure maintenance: application to water supply systems, Journal of Decision Systems 8 (1999) 116.

[18] T. Hadzilacos, D. Kalles, N. Preston, P. Melbourne, L. Camarinopoulos, M. Eimermacher, V. Kallidromitis, S. Frondistou-Yannas, S. Saegrov, UtilNets: water mains rehabilitation decision-support system, Computers Environment and Urban Systems 24 (2000) 215-232.

[19] K.D. Wong, Construction integrated management system for contractors, Journal of Buildings and Construction Management 8 (1) (2003) 12-18.

[20] K.D. Wong, The development of a web-based digital nervous system for construction project management, Proceedings of CRIOCM 2004 International Research Symposium on Advancement of Construction Management and Real Estate, Hong-Kong, 2004, pp. 115-118.

[21] K.D. Wong, The application of a computerized financial control system for the decision support of target cost contracts, ITcon, 11, Special Issue Decision Support Systems for Infrastructure management, 2006, pp. 257-268.

[22] A. Salatian, B. Taylor, Abstractor: an agglomerative approach to interpreting building monitoring data, ITcon 13 (2008) 193.

[23] I. Flood, Towards the next generation of artificial neural networks for civil engineering, Advanced Engineering in Informatics 22 (1) (2006) 414.

[24] D.E. Grierson, Pareto multi-criteria decision making, Advanced Engineering in Informatics 22 (3) (2008) 371-384.

[25] SETRA, IQOA (Image de la Qualité des Ouvrages d'Art), Guide de visite en subdivision, Référence F 9630 PV, 1996, Bureau de vente du SETRA

[26] J.F. Sageau, Sites, Risque et Génie Civil, Le Suivi préventif des structures, Colloque Paris Unesco, Paris, 2000, pp. 541-552.

[27] J.T. Smith, S.L. Tighe, The analytical hierarchy process as a tool for infrastructure management, in: 1st Annual Inter-university symposium on infrastructure management, Waterloo, ON, Canada, 2006.

[28] T.L. Saaty, The Analytic Hierarchy Process, McGraw-Hill, New York, 1980.

[29] B. Nyström, P. Söderholm, Selection of maintenance actions using the analytic hierarchy process (AHP): decision-making in railway infrastructure, Structure and Infrastructure Engineering, 5 (1) (2009), in press. 
[30] E. Chan, H. Suen, C. Chan, MAUT-based dispute resolution selection model prototype for international construction projects, Journal of Construction Engineering and Management 132 (5) (2006) 444-451.

[31] A.Y. Ababutain, A multi criteria decision-making model for selection of bot toll road proposals within the public sector, PhD Thesis, University of Pittsburgh, USA, 2001.

[32] J. Montmain, A. Akharraz, G. Mauris, Knowledge management as a support for collective decision-making and argumentation processes, in: IPMU'2002, 9th International Conference on Information processing and Management of uncertainty in Knowledge-Based Systems, Annecy, France, 2002.

[33] J. Montmain, G. Mauris, A. Akharraz, Elucidation and decisional risk in a mult criteria decision based on a Choquet integral aggregation - a cybernetic framework, International Journal of Multi-Criteria Decision Analysis 13 (5-6) (2005) 239-258.

[34] A. Denguir-Rekik, G. Mauris, J. Montmain, Propagation of uncertainty by the possibility theory in Choquet integral based decision making: application to an e-business website choice support, IEEE Transactions on Instrumentation and Measurement 55 (33) (2006) 721-728.

[35] S. Gentil, J. Montmain, Complex systems hierarchical representation supporting human decision-making, Advanced Engineering Informatics 18 (2005) 143-159.

[36] B.V. Dasarathy, Elucidative fusion systems: an exposition, Information Fusion 1 (2000) 5-15.

[37] P. Mora, A. Tsoukiàs, Decision Aiding and Argumentation, EUMAS 03 workshop, Oxford, England, 2003.

[38] C. Niculae, S. French, Bringing understanding in societal decision making: explaining and communicating analyses?, Journal of Multi-Criteria Decision Analysis $12(2-3)(2003)$ 191-202.

[39] A. Denguir-Rekik, G. Mauris, J. Montmain, A possibilistic-valued multi-criteria decision-making support for marketing activities in e-commerce: feedback based diagnosis system. European Journal of Operational Research, 195 (3) (2008) 876-888.

[40] E. Paschetta, A. Tsoukiàs, A real world MCDA application: evaluating software, Journal of Multi-Criteria Decision Analysis 9 (2000) 205-226.

[41] B. Roy, Paradigms and Challenges in MCDA, in: J. Figueira, S. Greco, M. Ehrgott (Eds.), Multiple Criteria Decision Analysis, Kluwer Academic Publishers, 2004 pp. 3-24.

[42] H.A. Simon, The New Science of Management Decision, Prentice Hall, New Jersey, 1977.

[43] H.A. Simon, The Science of Artificial, M.I.T. Press, Cambridge, 1982.

[44] H.A. Simon, Models of Bounded Rationality, MIT Press, Cambridge Massachusetts, vol. 3, 1977

[45] B. Roy, D. Bouyssou, Aide multicritère à la décision: méthodes et cas, Économica Paris, 1993.

[46] R. Slowinski, Fuzzy Sets in Decision Analysis, Operations Research and Statistics. The Handbook of Fuzzy Set, Kluwer Academic, 1998.

[47] L. Suchman, Plans and Situated Actions, Cambridge University Press, 1987.

[48] P. Trouillet, DRCA, Risque et Génie Civil, Suivi préventif des ponts autoroutiers du réseau concédé, Colloque Paris Unesco, Paris, 2000.
[49] C.A. Bana e Costa, J.C. Vansnick, MACBETH - an interactive path towards the construction of cardinal value functions, International Transactions in Operational Research 1 (1994) 489-500.

[50] V. Cliville, L. Berrah, G. Mauris, Quantitative expression and aggregation of performance measurements based on the MACBETH multi-criteria method, International Journal of Production Economics 105 (1) (2007) 171-189.

[51] D. Dubois, H. Prade, Criteria aggregation and ranking of alternatives in the framework of fuzzy set theory, Fuzzy Sets and Decision Analysis, TIMS Studies in the Management Sciences 20 (1984) 209-240.

[52] D. Dubois, H. Prade, A review of fuzzy set aggregation connectives, Information Sciences 36 (1985) 85-121.

[53] J.-L. Marichal, Aggregation operators for multi criteria decision aid. PhD thesis, Institute of Mathematics, Univ. Of Liège, Belgium, 1998.

[54] J. Montmain, S. Gentil, Operation support for alarm filtering, IEEE Conference CESA 96, Computational Engineering in systems applications, Lille, France, 1996.

[55] D.H. Krantz, R.D. Luce, P. Suppes, A. Tversky, Foundations of measurement, volume 1: Additive and Polynomial Representations. Academic Press, 1971.

[56] S. Jullien, G. Mauris, L. Valet, Ph. Bolon, Decision aiding tools for Animated film selection from a mean aggregation of criteria preferences over a finite scale, in: 11th Int. Conference on Information processing and Management of uncertainty in Knowledge-Based Systems, Paris, France, 2006.

[57] M. Grabisch, Representation of preferences over a finite scale by a mean operator, Mathematical Social Sciences 52 (2) (2006) 131-151.

[58] C. Sanchez, C. Spécification et implémentation du système d'aide à la décision multicritère pour la maintenance préventive et la gestion du patrimoine de la société d'autoroute ESCOTA: le projet SINERGIE, PhD thesis, Ecole des Mines de Paris, December 7th 2007, p. 198, 2007.

[59] A. Akharraz, J. Montmain, G. Mauris, A project decision support system based on an elucidative fusion system, in: Fusion 2002, 5th International Conference on Information Fusion, Annapolis, Maryland, USA, 2002

[60] M. Mavrovouniotis, G. Stephanopoulos, Formal order-of-magnitude reasoning in process engineering, Computer and Chemical Engineering $2(9 / 10)(1988)$ 867-880.

[61] C. Sanchez, J. Montmain, M. Vinches, B. Mahieu, A multi criteria evaluation over a finite scale for maintenance activities of a motorway operator. ICINCO 2007, in: 4th International Conference on Informatics in Control, Automation and Robotics, Angers, France, 2007.

[62] B. Roy, A missing Link in OR-DA, robustness analysis, Foundations of Computing and Decision Sciences 23 (3) (1998) 141-160.

[63] Ph. Vincke, Robust and neutral methods for aggregating preferences into an outranking relation, European Journal of Operational Research 112 (2) (1999) 405-412.

[64] Ph. Vincke, Robust solutions and methods in decision-aid, Journal of MultiCriteria Decision Analysis 8 (3) (1999) 181-187.

[65] L. Berrah, G. Mauris, J. Montmain, Diagnosis and improvement indexes for a multi-criteria industrial performance synthesized by a Choquet integral aggregation, The International Journal of Management Science, OMEGA 36 (3) (2008) 340-351. 